COMMUNICATIONS IN

ANALYSIS AND GEOMETRY

Volume 13, Number 2, 401-438, 2005

\title{
Moment Map, a Product Structure, and Riemannian Metrics with no Conjugate Points
}

\author{
Raúl M. Aguilar
}

Let $(\mathrm{M}, g$ ) be a complete Riemannian manifold, $G$ a group acting on $\mathrm{M}$ freely and properly by isometries with $\left(\mathrm{B}=\mathrm{M} / G, g_{\mathrm{B}}\right)$ its smooth Riemannian quotient.

We prove in Theorem 1 the uniqueness of a certain integrable structure on the tangent bundle of $\mathrm{M}$ defined in symplectic terms (2.1) and prove in Theorem 2 its naturality with respect to the symplectic reduction corresponding to the tangential action by $G$.

We define the notion of a "tangentially positive" isometric action and show in Theorem 3 how this condition implies that if $(\mathrm{M}, g)$ has no conjugate points its quotient $\left(\mathrm{B}, g_{\mathrm{B}}\right)$ has no conjugate points, and that the strongly stable and unstable distributions in the unit tangent bundle of $\mathrm{M}$ are natural under symplectic reduction, by our Theorem 4. In particular, we obtain conditions under which having a geodesic flow of Anosov type is inherited by the Riemannian quotient.

This work is followed up by [1] where we prove the converse of Theorem 3 and obtain some curvature restrictions for actions with conjugate point-free quotients.

\section{Introduction.}

Let $(\mathrm{M}, g)$ be a complete Riemannian manifold and $\pi: T \mathrm{M} \rightarrow \mathrm{M}$ its tangent bundle. The assignment $T \mathrm{M} \ni z \rightarrow(\exp (-z), \exp (z)) \in \mathrm{M} \times \mathrm{M}$ defines local "product coordinates" in a neighborhood of $\mathrm{M} \subset T \mathrm{M}$ corresponding to the two $\mathrm{M}$ factors. Infinitesimally it defines an integrable endomorphism $\mathbf{P}$ of $T(T \mathrm{M})$ with $\mathbf{P}^{2}=\mathbf{I}$ (the identity endomorphism) that interchanges the one-forms $\Theta$ and $d E$, where $E: T \mathrm{M} \rightarrow \mathbb{R}$ is the function $E(z)=\frac{1}{2} g(z, z)$ and $\Theta$ is the standard one-form defined by the metric and the tautological one-form in the cotangent bundle $T^{*} \mathrm{M}$.

In this paper we first show that in any neighborhood of the zero section of $T \mathrm{M}$ with the property of being serrate, meaning essentially the geodesic

\footnotetext{
${ }^{1}$ En honor del Instituto Balseiro en su Cinquentenario.
} 
convexity of its image by the map above, there is at most one such structure $\mathbf{P}$.

We then show that this characterization of $\mathbf{P}$ implies its naturality with respect to symplectic reduction for the tangential action induced by any group $G$ acting freely by isometries on $\mathrm{M}$ so that the quotient $\mathrm{M} / G$ is a smooth manifold. By the "naturality" of the reduction we mean that the following two conditions are satisfied:

1) There is a neighborhood of $\mathrm{M}$ in $T \mathrm{M}$ where $\mathbf{P}$ can be reduced;

2) the reduction of $\mathbf{P}$ coincides in some neighborhood of $\mathrm{B}=\mathrm{M} / G$ in $T \mathrm{~B}$ with the unique structure $\mathbf{P}_{\mathrm{B}}$ corresponding to the metric $g_{\mathrm{B}}$ that makes the projection $f:(\mathrm{M}) \rightarrow\left(\mathrm{B}, g_{\mathrm{B}}\right)$ a Riemannian submersion.

Condition 1 holds because the connection for the $G$-bundle $f: \mathrm{M} \rightarrow \mathrm{B}$ given by $g$ can be naturally extended to a connection in a portion of the total space of the $G$-bundle $\mu^{-1}(0) \rightarrow T \mathrm{~B}$ whose horizontal distribution is P-invariant, where $\mu: T \mathrm{M} \rightarrow \mathfrak{G}^{*}$ is the moment map of the tangential $G$ action and $\mathfrak{G}^{*}$ is the dual of the Lie algebra of $G$. In addition, since this connection is defined by $\mathbf{P}, G$ and especially the symplectic form $d \Theta$ on $T \mathrm{M}$, condition 2 holds also, as a consequence of the uniqueness result for $\mathbf{P}$ and the fact that the identification of $T B$ with the reduced space $\mu^{-1}(0) / G$ is symplectomorphic.

As an application we show that if $(\mathrm{M}, g)$ has no conjugate points, and the action satisfies the extra condition that the connection above can be defined on all of $\mu^{-1}(0)$, the quotient metric $g_{\mathrm{B}}$ on $\mathrm{B}$ has no conjugate points.

This additional condition for the action is expressed in terms of the positivity of the length of the action vector fields on $\mu^{-1}(0)$ with respect to the pseudo-Riemannian metric defined by $\mathbf{P}$ and the symplectic form $d \Theta$, and is referred to as the tangential positivity of the action.

When tangential positivity is satisfied on $\mu^{-1}(0)$, a reduction similar to that of $\mathbf{P}$ can be performed for re-scaled versions of $\mathbf{P}$, and in a certain limit of the re-scaling, the reduction corresponds to that of the distributions in the unit tangent bundle of $\mathrm{M}$ given by the strongly stable and unstable Jacobi fields; in this way we prove the naturality of those distributions under symplectic reduction. This is so because $\mathbf{P}$ is essentially a lift to $T \mathrm{M}$ of the shape operator of geodesic spheres on $\mathrm{M}$ which tends to the corresponding objects for horospheres; but we don't use this fact directly here.

There are situations where the triviality of the intersection of the strongly stable and unstable distributions in the unit tangent bundle is known to be equivalent to the property that the geodesic flow is of Anosov type; here the 
naturality under reduction of the stable and unstable distributions can be applied to derive conditions under which the geodesic flow in the quotient manifold is of Anosov type. We use results by P. Eberlein in [4] and derive a corollary along those lines.

The results above are trivially true for discrete groups $G$ since the quotient map $f: \mathrm{M} \rightarrow \mathrm{B}$ in this case is a Riemannian covering, in particular a local isometry. But, although such is the general situation for actions on compact manifolds with negative-definite Ricci curvature since their isometry groups are finite by a classical theorem of $\mathrm{S}$. Bochner, there is no such restriction on the dimension of groups of isometries of non-compact manifolds. However, constraints on isometric actions that are tangentially positive should in principle be expected, simply because the Riemannian quotient is curvature non-decreasing and thus it "promotes" the existence of conjugate points on $\left(\mathrm{B}, g_{\mathrm{B}}\right)$. In this paper we give as a sample restrictions for isometric actions on Euclidean space, which can be shown with what is developed here, and continue with further results in the forthcoming [1].

The paper is organized as follows: The main result in Section 2 is Theorem 1 where we prove the uniqueness of product structures on serrate sets satisfying the "symplectic condition" as defined by (2.1). Their existence is also proved in this section. Section 3 contains Theorem 2 where we show the naturality of the reduction of $\mathbf{P}$ for a proper and free isometric action as explained above. In section 4 we define what we mean by a tangentially positive action and show in Theorem 3 that the Riemannian submersion metric in $\mathrm{M} / G$ has no conjugate points if $\mathrm{M}$ has no conjugate points provided that the action is of that kind. In Section 5 Theorem 4 we show the naturality of the strongly stable and strongly unstable distributions on the unit tangent bundle with respect to reduction, and then derive two corollaries on Anosov type geodesic flows using work by P. Eberlein from [4] and W. Klingenberg from $[9]$.

Throughout the paper $(\mathrm{M}, g)$ will be a connected, smooth and complete Riemannian manifold of dimension $n$.

\section{Uniqueness of a certain structure on the tangent bundle.}

Let $\mathcal{O} \subset T \mathrm{M}$ be a neighborhood of $\mathrm{M}$, where $\mathrm{M}$ is identified with the zero section. An endomorphism $\mathbf{P}$ of the tangent bundle of $T \mathrm{M}$ such that $\mathbf{P}^{2}=\mathbf{I}$, the identity, and is integrable is called a (local) product structure in $\mathcal{O}$. Integrability of $\mathbf{P}$ means that for every point $z \in \mathcal{O}$ there are local "product

coordinates" $\left\{u_{1}^{-}, \cdots u_{n_{-}}^{-}, u_{1}^{+} \cdots, u_{n_{+}}^{-}\right\}$defined in some open set $\mathcal{U} \subset \mathcal{O}$ con- 
taining $z$, such that for all $v \in \mathcal{U}, T_{v}^{ \pm}(T \mathrm{M})=\operatorname{span}\left\{\frac{\partial}{\partial u_{1}^{ \pm}}, \cdots \frac{\partial}{\partial u_{n_{ \pm}}^{ \pm}}\right\}$, where $\left.T(T \mathrm{M})\right|_{\mathcal{O}}=[T(T \mathrm{M})]^{+} \oplus[T(T \mathrm{M})]^{-}$is the decomposition in the \pm 1 -eigenbundles of $\mathbf{P}$, hence $n_{-}+n_{+}=\operatorname{dim} T(T \mathrm{M})=2 n$.

Let $\Theta$ be the one-form on $T \mathrm{M}$ determined by $\Theta(U)=g\left(\pi_{*} U, z\right)$ for all $U \in T_{z} \mathrm{M}$ and $E$ the "energy function" on $T(T \mathrm{M}), E(z)=\frac{1}{2} g(z, z)$.

Definition 2.1. Adapted product structure. A product structure $\mathbf{P}$ in $\mathcal{O} \subset$ $T \mathrm{M}$ is adapted iff.

$$
\mathbf{P} \Theta=d E
$$

Let $\Sigma$ be the geodesic spray and $\Xi$ the Liouville (radial) vector field on TM. Recall (see [3], [10]) the definition of horizontal and vertical lifts of a vector $u \in T_{x} \mathrm{M}$ at $z$ in $T_{x} \mathrm{M}$, respectively $(u)_{z}^{h}$ and $(u)_{z}^{v}$ both in $T_{z}(T \mathrm{M})$ and determined by

$$
\pi_{*}(u)_{z}^{h}=\mathbf{K}(u)_{z}^{v}=u, \quad \pi_{*}(u)_{z}^{v}=\mathbf{K}(u)_{z}^{h}=0,
$$

where $\mathbf{K}: T_{z}(T \mathrm{M}) \rightarrow T_{\pi(z)} \mathrm{M}$ is the connection map. With this notation the geodesic spray $\Sigma$ and the Liouville vector field $\Xi$ are

$$
\Sigma(z)=(z)_{z}^{h}, \quad \Xi(z)=(z)_{z}^{v} .
$$

Definition 2.2. $\underline{\text { Serrate set. }}$. We call a set $\mathcal{O} \subset T(\mathrm{M})$ serrate if it is invariant by the contracting diffeomorphisms generated by $-\Sigma-\Xi$ and $\Sigma-\Xi$. In other words, $\mathcal{O}$ is characterized by the following property: if $\gamma$ is any unitspeed geodesic such that $y \dot{\gamma}(x) \in \mathcal{O}$ for some $x \in \mathbb{R}$ and some $y \in \mathbb{R}^{+}$then $\{(y-t) \dot{\gamma}(x+t),(y-t) \dot{\gamma}(x-t) \mid t \in[0, y]\} \subset \mathcal{O}$. (See Remark 2.1.)

Theorem 1. Uniqueness. In a serrate open $\mathcal{O} \subset T \mathrm{M}$ containing $\mathrm{M}$ there is at most one adapted product structure $\mathbf{P}$.

The proof of Theorem 1 is derived from the next several propositions and follows Corollary 2.4.1, with the proof that such $\mathbf{P}$ always exits right afterwards.

Proposition 2.1. An adapted product structure $\mathbf{P}$ defined on an open $\mathcal{O} \subset$ $T(T \mathrm{M})$ satisfies $\mathbf{P} \Sigma=\Xi$ on $\mathcal{O}$.

Proof. From $\mathbf{P} d E=\Theta$ we have $d(\mathbf{P} d E)=d \Theta$, and, since $\mathbf{P}$ is integrable, in local product coordinates there are functions $t_{i k}$ so that $d \Theta=$ 
$\sum_{i=1}^{n_{-}} \sum_{k=1}^{n_{+}} t_{i k} d u_{i}^{-} \wedge d u_{j}^{+}$. This implies that $d \Theta$ is anti-P-invariant, that is, for all $z \in \mathcal{O}$ and all $U$ and $V$ in $T_{z}(T \mathrm{M})$,

$$
d \Theta(\mathbf{P} U, \mathbf{P} V)=-d \Theta(U, V) .
$$

Now $d \Theta(U, V)=g\left(\mathbf{K} U, \pi_{*} V\right)-g\left(\pi_{*} U, \mathbf{K} V\right)$, hence $\Sigma$ is the Hamiltonian vector field for $E$, that is for all $U \in T_{z}(T \mathrm{M}), d E(U)=g(\mathbf{K} U, z)=$ $d \Theta(U, \Sigma)$. Similarly we have $\Theta(U)=g\left(\pi_{*} U, z\right)=d \Theta(\Xi, U)$. Thus, for arbitrary $U \in T_{z}(T \mathrm{M})$,

$$
d \Theta(\Xi, U)=\Theta(U) \stackrel{(i)}{=} d E(\mathbf{P} U)=d \Theta(\mathbf{P} U, \Sigma) \stackrel{(i i)}{=} d \Theta(\mathbf{P} \Sigma, U),
$$

where (i) is $\mathbf{P} d E=\Theta$ while (ii) the anti-P-invariance of $d \Theta$ together with its anti-symmetry. So $\Xi=\mathbf{P} \Sigma$, since $d \Theta$ is non-degenerate.

Corollary 2.1.1. $\operatorname{dim}\left[\left.T(T \mathrm{M})\right|_{\mathcal{O}}\right]^{ \pm}=n$.

Proof. This follows from the non-degeneracy of $d \Theta$ and its anti-P-invariance which implies that $d \Theta(U, V)=0$ if $U$ and $V$ are both eigen-vectors with the same +1 or -1 eigenvalue.

Proposition 2.2. Let $p \in \mathrm{M}, u \in T_{p} \mathrm{M}$ and $0_{p}$ the origin of $T_{p} \mathrm{M}$. An adapted product $\mathbf{P}$ structure on an open set $\mathcal{O} \supset \mathrm{M}$ must satisfy

$$
\mathbf{P}(u)_{0_{p}}^{h}=(u)_{0_{p}}^{v} .
$$

Proof. From Proposition 2.1 $\mathbf{P} \Sigma=\Xi$ which by (2.3) reads on $T \mathrm{M} \backslash \mathrm{M}$,

$$
\mathbf{P}(z)_{z}^{h}=(z)_{z}^{v}
$$

We multiply the left hand side of (2.6) by $t>0$ along the fibers of $T(T \mathrm{M}) \rightarrow T \mathrm{M}$ and along the fibers of $T \mathrm{M} \rightarrow \mathrm{M}$ as appropriate to get $t\left(\mathbf{P} z_{z}^{h}\right)=\mathbf{P} t\left(z_{z}^{h}\right) \stackrel{(*)}{=} \mathbf{P}(t z)_{z}^{h}$, where equality $(*)$ holds because $t\left(z_{z}^{h}\right)-(t z)_{z}^{h}$ is in the kernel of both $\mathbf{K}$ and $\pi_{*}$, hence is equal to zero. Similarly, we multiply the right-hand side of $(2.6)$ to get $t\left(z_{z}^{v}\right)=(t z)_{z}^{v}$.

It follows that $\mathbf{P}(t z)_{z}^{h}=(t z)_{z}^{v}$, and, by replacing $z$ by $\epsilon z$ with $\epsilon=t^{-1}$, we get

$$
\mathbf{P}(z)_{\epsilon z}^{h}=(z)_{\epsilon z}^{v} .
$$

Taking $\epsilon \mapsto 0$, by continuity of $\mathbf{P}$ and of the horizontal and vertical distributions on $T \mathrm{M}$ determined by the connection, we have, $\mathbf{P}(z)_{0_{p}}^{h}=(z)_{0_{p}}^{v}$. 
Corollary 2.2.1. Give $T \mathbb{R}=\mathbb{R}^{2}$ the structure $\mathbf{P}_{0} \frac{\partial}{\partial x}=\frac{\partial}{\partial y}$, where $(x, y) \in$ $\mathbb{R}^{2}$ is identified with $\left.y \frac{\partial}{\partial x}\right|_{x} \in T \mathbb{R}$; let $\mathbf{P}$ be an adapted product structure on $\mathcal{O} \subset T \mathrm{M}$ with $\mathrm{M} \subset \mathcal{O}$. For any unit-length geodesic $\gamma: \mathbb{R} \rightarrow \mathrm{M}, \gamma_{*}: T \mathbb{R} \rightarrow$ TM preserves the product structures:

$$
\left(\gamma_{*}\right)_{*} \circ \mathbf{P}_{0}=\mathbf{P} \circ\left(\gamma_{*}\right)_{*} .
$$

Proof. With the given parametrization of $T \mathbb{R}$, let $z=\gamma_{*}(x, y)=y \dot{\gamma}(x) \in$ TM.

The path $t \mapsto y \dot{\gamma}(x+t) \in T \mathrm{M}$ is horizontal (in $T \mathrm{M}$ ) with respect to the Levi-Civita connection on $\mathrm{M}$, goes through $z$ at $t=0$, and its projection $t \mapsto \gamma(x+t) \in \mathrm{M}$ has velocity $\dot{\gamma}(x)$ at $t=0$. Thus, by definition of horizontal lift,

$$
\left(\gamma_{*}\right)_{*}\left(\left.\frac{\partial}{\partial x}\right|_{(x, y)}\right)=\left.\frac{\partial}{\partial t}\right|_{t=0}(y \dot{\gamma}(x+t))=(\dot{\gamma}(x))_{z}^{h \stackrel{(\dagger)}{=}} \mathbf{P}(\dot{\gamma}(x))_{z}^{v},
$$

where $(\dagger)$ follows from Proposition 2.1 if $y \neq 0$ and from Proposition 2.2 if $y=0$.

On the other hand $t \mapsto(y+t) \dot{\gamma}(x) \in T_{\gamma(x)} \mathrm{M}$ is a path that projects to the point $\gamma(x) \in \mathrm{M}$ with conditions so that

$$
\left(\gamma_{*}\right)_{*}\left(\left.\mathbf{P}_{0} \frac{\partial}{\partial x}\right|_{(x, y)}\right)=\left(\gamma_{*}\right)_{*}\left(\left.\frac{\partial}{\partial y}\right|_{(x, y)}\right)=\left.\frac{\partial}{\partial t}\right|_{t=0}((y+t) \dot{\gamma}(x))=(\dot{\gamma}(x))_{z}^{v} .
$$

Equality (2.7) follows from (2.8) and (2.9).

Remark 2.1. Let $0<r \in \mathbb{R}$. The set $T^{r} \mathrm{M}=\left\{z \in T \mathrm{M} \mid 2 g(z, z)<r^{2}\right\}$, is an example of a serrate neighborhood of $\mathrm{M}$, but the concept is more general. For instance, let

$E_{0}=\{x+y<1\} \cap\{x-y<1\} \cap\{-x+y<1\} \cap\{-x-y<1\} \subset \mathbb{R}^{2} \cong T \mathbb{R}$,

with $\mathbb{R}=\{y=0\}$ Euclidean. For an integer $n \neq 0$ let $E_{n}$ be the translate of $E_{0}$ in the $x$ direction, to the right if $n>0$ or to the left if $n<0$, an amount of $\sum_{k=0}^{|n|}(|n|-k) 2^{-k}$ units. $\mathcal{O}=\bigcup_{n} E_{n}$ is a serrate neighborhood of $\mathbb{R}$ that doesn't contain $T^{r} \mathbb{R}$ for any $r>0$.

Let $0 \neq z$ and $\gamma$ the unit speed geodesic with $\|z\| \dot{\gamma}(0)=z$. Let $c:[-a, a] \rightarrow$ $T \mathrm{M} \backslash \mathrm{M}$ for $0<a \in \mathbb{R}$ be a path in $T \mathrm{M}$ with $c(0)=z$, and for $|\lambda|<a$, let $\gamma^{\lambda}$ be the geodesic defined by the initial conditions $\dot{\gamma}^{\lambda}(0)=c(\lambda)$. The map

$$
\Psi(\lambda, x, y)=y \Phi_{x} c(\lambda)
$$


where $\Phi: \mathbb{R} \times T M$ is the geodesic flow, defines a variation of the map $\gamma_{*}$ through the family of maps $\gamma_{*}^{\lambda}$. The variational vector field

$$
\left.\tilde{J} \stackrel{\text { def }}{=} \Psi_{*} \frac{\partial}{\partial \lambda}\right|_{(0, x, y)} \in \Gamma\left(T \mathbb{R},\left(\gamma_{*}\right)^{-1} T(T \mathrm{M})\right)
$$

is a vector field along the Riemannian leaf $\mathcal{L}_{\gamma}$ through $\gamma$,

$$
\mathcal{L}_{\gamma} \stackrel{\text { def }}{=}\left\{y \dot{\gamma}(x) \in T \mathrm{M} \mid(x, y) \in \mathbb{R}^{2}\right\},
$$

that restricts to a Jacobi field $J$ along $\gamma$.

Proposition 2.3. If $\mathcal{L}_{\gamma}$ is parametrized $(x, y) \mapsto z=y \dot{\gamma}(x) \in \mathcal{L}_{\gamma}$ the Jacobi field $\tilde{J}$ along $\mathcal{L}_{\gamma}$ can be expressed

$$
\tilde{J}(x, y)=(J(x))_{z}^{h}+\left(\nabla_{z} J(x)\right)_{z}^{v} .
$$

Proof. We have $\pi(\Psi(\lambda, x, y))=\pi\left(y \dot{\gamma}^{\lambda}(x)\right)=\gamma^{\lambda}(x)$. Now, the expression (2.12) follows from (2.2) and the calculations below:

$$
\pi_{*}(\tilde{J}(x, y))=\left.\frac{\partial}{\partial \lambda}(\pi \circ \Psi)\right|_{(0, x, y)}=\left.\frac{\partial}{\partial \lambda}\left(\gamma^{\lambda}(x)\right)\right|_{(0, x, y)}=J(x),
$$

and

$$
\mathbf{K}(\tilde{J}(x, y))=\left.\mathbf{K}\left(\frac{\partial \Psi}{\partial \lambda}\right)\right|_{(0, x, y)}=\left.\nabla_{\frac{\partial(\pi \circ \Psi)}{\partial \lambda}}(\Psi(\lambda, x, y))\right|_{(0, x, y)}=(\dagger),
$$

where, fixing $y$, viewing $(\lambda, x) \mapsto(\pi \circ \Psi)(\lambda, x, y)=\gamma^{\lambda}(x)$ as a variation, and using that the connection $\nabla$ is torsion-free,

$$
\begin{aligned}
(\dagger) & =\left.\nabla_{\frac{\partial(\pi \circ \Psi)}{\partial \lambda}}\left(y \frac{\partial(\pi \circ \Psi)}{\partial x}\right)\right|_{(0, x, y)} \\
& =\left.y \nabla_{\frac{\partial(\pi \circ \Psi)}{\partial x}}\left(\frac{\partial(\pi \circ \Psi)}{\partial \lambda}\right)\right|_{(0, x, y)}=y \nabla_{\dot{\gamma}(x)} J=\nabla_{z} J
\end{aligned}
$$

We refer to $\tilde{J}$ given by (2.12) as the "canonical extension" of $J$ along the Riemannian leaf $\mathcal{L}_{\gamma}$.

Proposition 2.4. Let $\mathcal{O}$ be a serrate neighborhood of $\mathrm{M}$ where an adapted product structure $\mathbf{P}$ is defined. Let $\gamma$ be a unit-speed geodesic, $J$ a Jacobi field along $\gamma$ and $\tilde{J}$ its canonical extension along $\mathcal{L}_{\gamma}$. If $y \dot{\gamma}(x) \in \mathcal{O}$ where $y>0$, then the following are equivalent: 
i) $J(x+y)=0 \quad($ resp. $J(x-y)=0)$,

ii) $[\tilde{J}(x, y)]^{+}=0 \quad\left(\operatorname{resp} .[\tilde{J}(x, y)]^{-}=0\right)$,

iii) $[\tilde{J}(x+t, y-t)]^{+}=0\left(\operatorname{resp} .[\tilde{J}(x-t, y-t)]^{-}=0\right) \forall t \in[0, y]$,

where we used $[U]^{ \pm}=\frac{1}{2}(U \pm \mathbf{P} U)$.

Proof. Given $z=y \dot{\gamma}(x)$ in $\mathcal{O}$ let $\left\{u_{1}^{-}, \cdots, u_{n}^{-}, u_{1}^{+}, \cdots, u_{n}^{+}\right\}$be a product coordinate chart defined in some neighborhood $\mathcal{U} \subset \mathcal{O}$ containing the path $\lambda \mapsto y \dot{\gamma}^{\lambda}(x)$ for $\lambda \in\left[-a^{\prime}, a^{\prime}\right]$ with $a^{\prime}>0$ small enough.

By Corollary 2.2.1 for each $\lambda$ the map $\gamma_{*}^{\lambda}:\left(\gamma_{*}^{\lambda}\right)^{-1}(\mathcal{O}) \rightarrow T \mathcal{O}$ preserves the product structures, $\mathbf{P}_{0}$ in $T \mathbb{R}$ and $\mathbf{P}$ on $\mathcal{O}$. It follows that for all $1 \leq i \leq n$

$$
\frac{\partial}{\partial s^{-}}\left(u_{i}^{+}\left(y \dot{\gamma}^{\lambda}(x)\right)=0, \quad \frac{\partial}{\partial s^{+}}\left(u_{i}^{-}\left(y \dot{\gamma}^{\lambda}(x)\right)=0\right.\right.
$$

where $s^{-}=x-y$ and $s^{+}=x+y$ are global product coordinates for $\mathbf{P}_{0}$ in $T \mathbb{R} \cong \mathbb{R}^{2}$.

Hence, by differentiation of the map $\Psi$ (2.10) with respect to $\lambda$, there are one-variable functions $h_{i}^{+}$and $h_{i}^{-}$such that

$$
\tilde{J}(x, y)=\Psi_{*}\left(\left.\frac{\partial}{\partial \lambda}\right|_{(0, x, y)}\right)=\left.\sum_{i=1}^{n} h_{i}^{+}(x+y) \frac{\partial}{\partial u_{i}^{+}}\right|_{y \dot{\gamma}(x)}+\left.h_{i}^{-}(x-y) \frac{\partial}{\partial u_{i}^{-}}\right|_{y \dot{\gamma}(x)} .
$$

But if $\left\{v_{1}^{-}, \cdots, v_{n}^{-}, v_{1}^{+}, \cdots, v_{n}^{+}\right\}$is another product-coordinate chart about $y \dot{\gamma}^{\lambda}(x)$, we have $\tilde{J}(x, y)=\left.\sum_{i=1}^{n} k_{i}^{+}(x+y) \frac{\partial}{\partial v_{i}^{+}}\right|_{y \dot{\gamma}(x)}+\left.k_{i}^{-}(x-y) \frac{\partial}{\partial v_{i}^{-}}\right|_{y \dot{\gamma}(x)}$, where necessarily $h_{i}^{ \pm}(x \pm y)=\sum_{j=1}^{n} k_{j}^{ \pm}(x \pm y) \frac{\partial u_{i}^{ \pm}}{\partial v_{j}^{ \pm}}$.

It follows that the condition $[\tilde{J}(x, y)]^{+}=0 \quad\left(\operatorname{resp} .[\tilde{J}(x, y)]^{-}=0\right)$ is open and closed in the connected subset of $\mathcal{O}$

$$
\{(y-t) \dot{\gamma}(x+t) \mid t \in[0, y]\} \quad(\operatorname{resp} . \quad\{(y-t) \dot{\gamma}(x-t) \mid t \in[0, y]\}),
$$

the connectedness implied by the hypothesis that $\mathcal{O}$ is serrate.

The argument above proves $i i) \Longleftrightarrow i i i$ ). It also proves $i) \Longleftrightarrow i i i)$ once we note that given $s \in \mathbb{R}$ we have

$$
\begin{aligned}
0 & =[\tilde{J}(s, 0)]^{ \pm} \underset{(a)}{=}\left[(J(s))_{0_{\gamma(s)}}^{h}\right]^{ \pm} \underset{(b)}{=} \frac{1}{2}\left((J(s))_{0_{\gamma(s)}}^{h} \pm(J(s))_{0_{\gamma(s)}}^{v}\right) \\
& \Longleftrightarrow J(s)=0,
\end{aligned}
$$


where we used (2.12) in (a), Proposition 2.2 in (b), and the fact that horizontal and vertical lifts are linearly independent.

Corollary 2.4.1. Let $0 \neq z \in \mathcal{O}$, with $\mathcal{O}$ serrate where an adapted product structure $\mathbf{P}$ is defined. Let $\gamma$ be the unit-speed geodesic such that $z=y \dot{\gamma}(0)$ with $y>0$. Then, there are no pair of conjugate points of $\gamma$ contained in the segment $\gamma([-y, y])$.

Proof. We will get a contradiction from the assumption that $\gamma(a)=p$ and $\gamma(b)=q$ is a pair of conjugate points of $\gamma$ with $-y \leq a<b \leq y$. So, let $J$ be a non-zero Jacobi field along $\gamma$ such that $J(a)=0$ and $J(b)=0$. With the notation as in Corollary 2.2.1, because $\mathcal{O}$ is serrate and contains $(0, y)=\gamma_{*}^{-1}(z)$, the image under $\gamma_{*}$ of the triangular region in $\mathbb{R}^{2}$ with vertices $(-y, 0),(y, 0)$ and $(0, y)$ is contained in $\mathcal{O}$. In particular, the images under $\gamma_{*}$ of the straight segment from $(a, 0) \in \mathbb{R}^{2}$ to $\left(\frac{a+b}{2}, \frac{b-a}{2}\right) \in \mathbb{R}^{2}$ and of the segment from $(b, 0) \in \mathbb{R}^{2}$ to $\left(\frac{a+b}{2}, \frac{b-a}{2}\right) \in \mathbb{R}^{2}$ are contained in $\mathcal{O}$. Hence the extension $\tilde{J}(x, y)$ of $J$ to $\mathcal{L}_{\gamma} \cap \mathcal{O}$ satisfies

$$
\left[\tilde{J}\left(\frac{a+b}{2}, \frac{b-a}{2}\right)\right]^{+}=\left[\tilde{J}\left(\frac{a+b}{2}, \frac{b-a}{2}\right)\right]^{-}=0,
$$

which implies that $\mathbf{P} \tilde{J}\left(\frac{a+b}{2}, \frac{b-a}{2}\right)=0$. Then, since $\mathbf{P}$ is non-degenerate, we must have $\tilde{J}\left(\frac{a+b}{2}, \frac{b-a}{2}\right)=0$. Hence, by Proposition 2.4, $J\left(\frac{a+b}{2}\right)=0$ and $\nabla_{\dot{\gamma}\left(\frac{a+b}{2}\right)} J\left(\frac{a+b}{2}\right)=0$, and thus $J(x)=0$ for all $x$, which contradicts that $J$ is a non-zero Jacobi field.

2.0.1. Proof of Theorem 1. Let $\mathcal{O}$ be a serrate neighborhood of $\mathrm{M}$ where an adapted product structure $\mathbf{P}$ is defined. We will show that $\mathbf{P}$ is determined uniquely by the Riemannian metric $g$.

From Proposition 2.2 along the zero section $\mathbf{P}$ is uniquely defined by (2.5). So let $0 \neq z \in \mathcal{O}$. Let $\gamma$ be the unit-speed geodesic such that $z=y \dot{\gamma}(0)$ with $y>0$ and let $\left\{u_{1}, \cdots, u_{n}\right\}$ be an orthonormal basis of $T_{\gamma(0)} \mathrm{M}$.

Since $\mathcal{O}$ is serrate, by Corollary 2.4.1 there are no pair of conjugate points of $\gamma$ contained in the segment $\gamma([-y, y])$. In particular $\gamma(-y)$ and $\gamma(0)$ are not conjugate points of $\gamma$, and thus, there are Jacobi fields $\left\{J_{1}, \cdots, J_{n}\right\}$ along $\gamma$ such that for all $1 \leq i \leq n$ :

$$
J_{i}(-y)=0, \quad J_{i}(0)=u_{i}
$$


Similarly, $\gamma(0)$ and $\gamma(y)$ are not conjugate points of $\gamma$ and thus there are Jacobi fields $\left\{G_{1}, \cdots, G_{n}\right\}$ along $\gamma$ so that for all $1 \leq i \leq n$ :

$$
G_{i}(0)=u_{i}, \quad G_{i}(y)=0 .
$$

Let $\tilde{J}_{i}$ and $\tilde{G}_{i}$ be the corresponding canonical extensions to $\mathcal{L}_{\gamma}$. With the parameterization of $\mathcal{L}_{\gamma}$ as earlier, given by $(x, y) \mapsto y \dot{\gamma}(x) \in \mathcal{L}_{\gamma}$, it follows from Proposition 2.4 applied respectively to $\left\{\tilde{J}_{i}\right\}$ and to $\left\{\tilde{G}_{i}\right\}$ that for all $1 \leq i \leq n$ :

$$
\left[\tilde{J}_{i}(0, y)\right]^{-}=0 ; \quad\left[\tilde{G}_{i}(0, y)\right]^{+}=0
$$

equivalently

$$
\tilde{J}_{i}(0, y) \in\left[T_{z}(T \mathrm{M})\right]^{+} ; \quad \tilde{G}_{i}(0, y) \in\left[T_{z}(T \mathrm{M})\right]^{-} .
$$

Thus, since the Jacobi fields are defined by the Riemannian metric, and their equation is linear, in order to show that the splitting $T_{z}(T \mathrm{M})=$ $\left[T_{z}(T \mathrm{M})\right]^{-} \oplus\left[T_{z}(T \mathrm{M})\right]^{+}$is determined by the Riemannian metric, due to (2.14) it suffices to show that each of the two sets in $T_{z}(T \mathrm{M})$ given by $\left\{\tilde{J}_{1}(0, y), \cdots, \tilde{J}_{n}(0, y)\right\}$ and $\left\{\tilde{G}_{1}(0, y), \cdots, \tilde{G}_{n}(0, y)\right\}$ have rank $n$. But, from equation (2.12) it follows that for any given constants $a_{i} \in \mathbb{R}$,

$$
\pi_{*}\left(\sum_{i=1}^{n} a_{i} \tilde{J}_{i}(0, y)\right)=\sum_{i=1}^{n} a_{i} J_{i}(0)=\sum_{i=1}^{n} a_{i} u_{i}
$$

and hence the $\left\{\tilde{J}_{i}(0, y)\right\}$ are linearly independent. Similarly for $\left\{\tilde{G}_{i}(0, y)\right\}$.

The argument above applied to all $0 \neq z \in \mathcal{O}$ shows the uniqueness of $\mathbf{P}$.

\subsubsection{Existence of the adapted product structure.}

Proposition 2.5. Let $(\mathrm{M}, g)$ be a complete Riemannian manifold. Then there is an open serrate $\mathcal{O} \subset T \mathrm{M}$ containing $\mathrm{M}$ where the adapted product structure is defined. Moreover, $\mathcal{O}$ can be taken to be the whole TM if and only if $(\mathrm{M}, g)$ has no conjugate points.

Proof. Let $F: T \mathrm{M} \rightarrow \mathrm{M} \times \mathrm{M}$ be given by $F(z)=(\exp (-z), \exp (z))=$ $\left(\pi\left(\Phi_{-1} z\right), \pi\left(\Phi_{1} z\right)\right)$, where $\Phi$ is the geodesic flow. The set

$$
\mathcal{O}_{F} \stackrel{\text { def }}{=}\left\{z \in T \mathrm{M} \mid \operatorname{rank} F_{*}=2 n\right\}
$$


contains the zero section since for all $p \in \mathrm{M}$ and all $v \in T_{p} \mathrm{M}$ it holds $F_{*}\left((v)_{0_{p}}^{h}\right)=(v, v)$ and $F_{*}\left((v)_{0_{p}}^{v}\right)=(-v, v)$, both in $T_{p} \mathrm{M} \oplus T_{p} \mathrm{M}$, and hence $F_{*}$ is non-singular along $\mathrm{M}$.

To show that there is an open serrate $\mathcal{O} \subset \mathcal{O}_{F}$ we need to describe $F_{*}$ off $\mathrm{M}$.

Proposition 2.6. Let $\gamma$ be a unit-speed geodesic and let $y>0$. The geodesic segment $\gamma([-y, y])$ contains no pair of conjugate points of $\gamma$ if and only if for all $a$ and $b$ in $\mathbb{R}$ with $-y \leq a<b \leq y$,

$$
\left(\frac{b-a}{2}\right) \dot{\gamma}\left(\frac{a+b}{2}\right) \in \mathcal{O}_{F} .
$$

Proof. Let $0 \neq z \in T \mathrm{M}$ and $U_{z} \in T_{z}(T \mathrm{M})$. Let $c:[-a, a] \rightarrow T \mathrm{M} \backslash \mathrm{M}$ be a path disjoint from the zero section such that $c(0)=z$ and $c_{*}\left(\left.\frac{\partial}{\partial \lambda}\right|_{\lambda=0}\right)=U$. We calculate $F_{*}\left(U_{z}\right)=\left.\frac{\partial}{\partial \lambda}\right|_{\lambda=0} F(c(\lambda))$, using

$$
\Phi_{s}(c(\lambda))=\|c(\lambda)\| \dot{\gamma}^{\lambda}(s\|c(\lambda)\|),
$$

where $\gamma^{\lambda}$ is the unit-speed geodesic with initial conditions $\dot{\gamma}^{\lambda}(0)=$ $\|c(\lambda)\|^{-1} c(\lambda)$. It follows that

$$
F_{*}\left(U_{z}\right)=(J(-\|z\|), J(\|z\|)) \in T_{x_{1}} \mathrm{M} \oplus T_{x_{2}} \mathrm{M},
$$

where $x_{1}=\pi\left(\Phi_{-1} z\right)=\exp _{\pi(z)}(-z)$ and $x_{2}=\pi\left(\Phi_{1} z\right)=\exp _{\pi(z)}(z)$, while $J(-\|z\|)$ and $J(\|z\|)$ are the evaluations at $x_{1}=\gamma(-\|z\|)$ and $x_{2}=\gamma(\|z\|)$ of the Jacobi field along the geodesic $\gamma=\gamma^{0}$ determined by the variation $(s, \lambda) \mapsto \Phi_{s} c(\lambda)$.

The previous argument shows that $0 \neq z \in \mathcal{O}_{F}$ if and only if along the unit-speed geodesic $\gamma$ defined by $\|z\| \dot{\gamma}(0)=z$ there is a unique Jacobi field whose values at $\gamma(-\|z\|)$ and $\gamma(\|z\|)$ can be arbitrarily prescribed, which is equivalent to $\gamma(-\|z\|)$ and $\gamma(\|z\|)$ not being a pair of conjugate points of $\gamma$. Thus, Proposition 2.6 is proved by taking $z$ as in (2.15) and noting that, from the identity $\Phi_{s}(t \dot{\gamma}(r))=t \dot{\gamma}(r+s t)$, it holds $F\left(\left(\frac{b-a}{2}\right) \dot{\gamma}\left(\frac{a+b}{2}\right)\right)=(\gamma(a), \gamma(b))$.

We can now define our serrate set $\mathcal{O} \subset \mathcal{O}_{F}$. It is a classical result that any complete Riemannian manifold $\mathrm{M}$ can be covered by open sets $U_{\alpha}, \mathrm{M}=\cup_{\alpha \in A} U_{\alpha}$, such that any pair of points $x_{1}$ and $x_{2}$ in $U_{\alpha}$ can be joined by a unique unit speed minimizing geodesic, call it $\gamma_{x_{1} x_{2}}$, with 
$\gamma_{x_{1} x_{2}}\left(-2^{-1} d\left(x_{1}, x_{2}\right)\right)=x_{1}$ and $\gamma_{x_{1} x_{2}}\left(2^{-1} d\left(x_{1}, x_{2}\right)\right)=x_{2}$, contained in $U_{\alpha}$, where $d\left(x_{1}, x_{2}\right)$ is the distance from $x_{1}$ to $x_{2}$. It follows that $\gamma_{x_{1} x_{2}}$ is minimizing for any pair of points in $\gamma_{x_{1} x_{2}}((-\infty,+\infty)) \cap U_{\alpha}$, which, as it is well-known, implies that there are no pairs of conjugate points of $\gamma_{x_{1} x_{2}}$ in $\gamma_{x_{1} x_{2}}((-\infty,+\infty)) \cap U_{\alpha}$. It follows that the set

$$
W_{\alpha} \stackrel{\text { def }}{=}\left\{2^{-1} d\left(x_{1}, x_{2}\right) \dot{\gamma}_{x_{1}, x_{2}}(0) \mid\left(x_{1}, x_{2}\right) \in U_{\alpha} \times U_{\alpha}\right\}
$$

is contained in $\subset \mathcal{O}_{F} \subset T \mathrm{M}$, and that it is open, since it is diffeomorphic to $U_{\alpha} \times U_{\alpha}$ under $F$. It also follows, using Proposition 2.6, that $W_{\alpha}$ is serrate. Thus, since the union of serrate sets is a serrate set, the open set

$$
\mathcal{O} \stackrel{\text { def }}{=} \cup_{\alpha \in A} W_{\alpha}
$$

is serrate and is contained in $\mathcal{O}_{F}$.

Thus, via $F$ we pull back the global product structure $\mathbf{P}^{\times}$of $\mathrm{M} \times \mathrm{M}$ to a local product structure $\mathbf{P}^{F}$ in $\mathcal{O} \subset \mathcal{O}_{F}$. Here $T(\mathrm{M} \times \mathrm{M}) \cong T \mathrm{M} \times T \mathrm{M}$, the right and left factors corresponding respectively to the integral manifolds for the -1 and +1 sub-bundles for $\mathbf{P}^{\times}$.

It remains to be shown that $\mathbf{P}^{F} \Theta=d E$, so that, by Theorem $1, \mathbf{P}^{F}$ is actually the adapted product structure $\mathbf{P}$. So, let $z$ in $\mathcal{O}_{F} \backslash \mathrm{M}$, and let $\gamma$ be the unit-speed geodesic with $y \dot{\gamma}(0)=z$ with $y>0$. The Jacobi field $J_{1}$ along $\gamma$ with initial conditions $J_{1}(0)=0$ and $\nabla_{z} J_{1}(0)=z$ is given by $J_{1}(s)=s \dot{\gamma}(s)$ for $s \in \mathbb{R}$. Thus, recalling $(2.16)$ and $\Xi(z)=(z)_{z}^{v}$,

$$
\mathbf{P}^{\times}\left(F_{*}(\Xi)\right)=\mathbf{P}^{\times}\left(J_{1}(-y), J_{1}(y)\right)=\left(-J_{1}(-y), J_{1}(y)\right)=(y \dot{\gamma}(-y), y \dot{\gamma}(y)) .
$$

On the other hand the Jacobi field $J_{2}$ along $\gamma$ with initial conditions $J_{2}(0)=$ $z$ and $\nabla_{z} J_{2}(0)=0$ is given by $J_{2}(x)=y \dot{\gamma}(s)$ for all $s \in \mathbb{R}$, and thus by (2.16) and $\Sigma(z)=(z)_{z}^{h}$,

$$
F_{*}(\Sigma)=\left(J_{2}(-y), J_{2}(y)\right)=(y \dot{\gamma}(-y), y \dot{\gamma}(y)) .
$$

It follows that on $\mathcal{O}, \mathbf{P}^{F}(\Xi)=F_{*}^{-1}\left(\mathbf{P}^{\times}\left(F_{*}(\Xi)\right)\right)=\Sigma$ and, from the nondegeneracy of $d \Theta$, that $\mathbf{P}^{F} \Theta=d E$.

Finally, if $(\mathrm{M}, g)$ has no conjugate points it is clear that $\mathcal{O}_{F}=T \mathrm{M}$, which is serrate.

\section{Naturality of $\mathbf{P}$ with respect to symplectic reduction.}

In this section we show that the adapted structure $\mathbf{P}$ is "natural" with respect to symplectic reduction in the following sense: 
1) In a neighborhood of $\mathrm{M}$ in $T \mathrm{M}$ a reduction of $\mathbf{P}$ is possible;

2) The product structure so induced in a neighborhood of $\mathrm{B}$ in $\mathrm{TB}$ agrees, in a perhaps smaller neighborhood of $\mathrm{B}$, with the adapted structure given by $g_{\mathrm{B}}$,

Part 1 follows from the existence of a connection with $\mathbf{P}$-invariant horizontal distribution in the $G$-bundle defined by the tangential action on the zero level set of the moment map in $T \mathrm{M}$, while part 2 relies on the fact that such connection is defined using the symplectic structure on $T \mathrm{M}$ which, as a consequence the very definition of Riemannian submersion metric for isometric actions, is itself natural under reduction.

3.0.3. Set up. (See [12].) Let $G$ be a closed subgroup of the group of isometries of $(\mathrm{M}, g)$ and $\mathfrak{G}$ its Lie algebra. The action $G \times \mathrm{M} \mapsto \mathrm{M}$ induces the action $G \times T \mathrm{M} \mapsto T \mathrm{M}$ by tangent maps which preserves the one-form $\Theta$, for $h \pi=\pi h_{*}$ and $h_{*} \pi_{*}=\pi_{*}\left(h_{*}\right)_{*}$ hence for all $U \in T_{z}(T \mathrm{M})$ we have, $\left(h_{*}\right)^{*} \Theta(U)=\Theta\left(\left(h_{*}\right)_{*} U\right)=g\left(\pi_{*}\left(h_{*}\right)_{*} U, h_{*} z\right)=g\left(h_{*} \pi_{*} U, h_{*} z\right)$, which is equal to $g\left(\pi_{*} U, z\right)=\Theta(U)$ if $h$ is an isometry.

Now, given $\xi \in \mathfrak{G}$ let $\xi_{\mathrm{M}}: \mathrm{M} \rightarrow T \mathrm{M}$ and $\xi_{T \mathrm{M}}: T \mathrm{M} \rightarrow T(T \mathrm{M})$ be the action vector fields on $\mathrm{M}$ and $T \mathrm{M}$ respectively. We denote by $\mathfrak{G}^{\mathrm{M}}$ and $\mathfrak{G}^{T \mathrm{M}}$ the corresponding distributions, that is, for $p \in \mathrm{M}$ and $z \in T \mathrm{M}$,

$$
\mathfrak{G}_{p}^{\mathrm{M}}=\left\{\xi_{\mathrm{M}}(p) \mid \xi \in \mathfrak{G}\right\}, \quad \mathfrak{G}_{z}^{T \mathrm{M}}=\left\{\xi_{T \mathrm{M}}(z) \mid \xi \in \mathfrak{G}\right\} \subset T_{z}(T \mathrm{M}) .
$$

Let $\mu: T \mathrm{M} \rightarrow \mathfrak{G}^{*}$ be the moment map of the tangent action, where $\mathfrak{G}^{*}$ is the dual of the Lie algebra of $G$. At $z \in T \mathrm{M}$ we have $\mu(z)[\xi]=\Theta\left(\xi_{T \mathrm{M}}(z)\right)=$ $g\left(z, \pi_{*}\left(\xi_{T \mathrm{M}}(z)\right)\right)=g\left(z, \xi_{\mathrm{M}}(\pi(z))\right)$ and thus

$$
\mu^{-1}(0)=\left\{z \in T \mathrm{M} \mid g\left(z, \xi_{\mathrm{M}}(\pi(z))\right)=0 \text { for all } \xi \in \mathfrak{G}\right\} .
$$

Evaluation of $\mu$ at a given $\xi \in \mathfrak{G}$ yields a function $\mu[\xi]: T \mathrm{M} \rightarrow \mathbb{R}$, and we have $0=\mathcal{L}_{\xi_{T \mathrm{M}}} \Theta=d(\mu[\xi])+d \Theta\left(\xi_{T \mathrm{M}}, \cdot\right)$, where the vanishing of the Lie derivative of $\Theta$ is the $G$-invariance explained in the first paragraph, and the second equality results from the standard Cartan formula. It follows that given $z \in \mu^{-1}(0)$ (see [12])

$$
T_{z}\left(\mu^{-1}(0)\right)=\left(\mathfrak{G}_{z}^{T \mathrm{M}}\right)^{\perp} \supset \mathfrak{G}_{z}^{T \mathrm{M}},
$$

the inclusion valid since $G$ acts on $\mu^{-1}(0), \Theta$ and the distribution $\mathfrak{G}^{T \mathrm{M}}$ being $G$-invariant, where, here and in the rest of the paper, given a subspace $A \subset T_{z}(T \mathrm{M})$ we put

$$
A^{\perp} \stackrel{\text { def }}{=}\left\{U \in T_{z}(T \mathrm{M}) \mid d \Theta(U, V)=0 \text { for all } V \in A\right\} .
$$


Since the $G$-action on $\mathrm{M}$ is free, the quotient map

$$
f: \mathrm{M} \rightarrow \mathrm{B}=\mathrm{M} / G
$$

is a principal $G$-bundle, with an $f$-horizontal distribution defined as the $g$ orthogonal complement of the fibers of $f$, which is precisely $\mu^{-1}(0) \subset T \mathrm{M}$. But $\pi_{*}\left(\xi_{T \mathrm{M}}(z)\right)=\xi_{\mathrm{M}}(\pi(z)) \neq 0$, hence the $G$-action on $\mu^{-1}(0)$ is also free and defines a principal $G$-bundle

$$
\mathfrak{p}: \mu^{-1}(0) \rightarrow \mu^{-1}(0) / G
$$

with $\mathfrak{p}=f_{*} \circ i_{\mu}$ where $i_{\mu}: \mu^{-1}(0) \hookrightarrow T \mathrm{M}$ is the inclusion.

There are induced on $\mu^{-1}(0) / G$ a function $\check{E}$ and a one-form $\check{\Theta}$ defined by

$$
\mathfrak{p}^{*} \check{E}=\left(i_{\mu}\right)^{*} E, \quad \mathfrak{p}^{*} \check{\Theta}=\left(i_{\mu}\right)^{*} \Theta,
$$

where $E: T \mathrm{M} \rightarrow \mathbb{R}, E(z)=\frac{1}{2} g(z, z)$. The form $\check{\Theta}$ is well-defined because $\Theta\left(\xi_{T \mathrm{M}}^{i}\right)=0$ in $\mu^{-1}(0)$, and $d \check{\Theta}^{2}$ is non-degenerate, since on $\mu^{-1}(0)$, by (3.1), $T\left(\mu^{-1}(0)\right) \cap\left(T\left(\mu^{-1}(0)\right)\right)^{\perp}=\left(\mathfrak{G}^{T \mathrm{M}}\right)^{\perp} \cap \mathfrak{G}^{T \mathrm{M}}=\mathfrak{G}^{T \mathrm{M}}=\operatorname{ker} \mathfrak{p}_{*}$.

If we identify the $G$-orbit of each $z \in \mu^{-1}(0)$ with $f_{*} z \in T_{f(\pi(z))}$ B we get,

$$
\mu^{-1}(0) / G \simeq T(\mathrm{M} / G)=T \mathrm{~B},
$$

and if we give $\mathrm{B}$ the Riemannian metric $g_{\mathrm{B}}$ that makes $f: \mathrm{M} \rightarrow \mathrm{B}$ a Riemannian submersion,

$$
\check{E}=E_{\mathrm{B}}, \quad \check{\Theta}=\Theta_{\mathrm{B}}
$$

where $\Theta_{\mathrm{B}}$ and $E_{\mathrm{B}}$ are the corresponding objects on $T \mathrm{~B}$ induced by the metric $g_{\mathrm{B}}$.

We now state the naturality of $\mathbf{P}$ with respect to reduction.

Theorem 2. Let $G$ act on $(\mathrm{M}, g)$ by isometries freely and properly. Let $\mathbf{P}$ be defined in an open $\mathcal{O} \subset T \mathrm{M}$ containing $\mathrm{M}$ where $\mathcal{O}$ is $G$-invariant (which is always possible by Proposition 3.1). Then, there is a $G$-invariant open set $\mathcal{O}^{\dagger} \subset \mathcal{O}$ containing $\mathrm{M}$ where $\mathbf{P}$ pushes down to a product structure $\check{\mathbf{P}}$ on the neighborhood of $B, \mathfrak{p}\left(\mu^{-1}(0) \cap \mathcal{O}^{\dagger}\right) \subset T$ B. Such $\check{\mathbf{P}}$ agrees in a perhaps smaller neighborhood of $\mathrm{B}$ in $\mathrm{TB}$ with the adapted product structure $\mathbf{P}_{\mathrm{B}}$ for the submersion metric $g_{\mathrm{B}}$.

The proof of Theorem 2 will be derived from Propositions (3.1) through (3.5) and follows Corollary 3.5.1. 
Proposition 3.1. Given any open $N \subset T \mathrm{M}$ containing $\mathrm{M}$ we can find a serrate open $\mathcal{O} \subset N$ containing $\mathrm{M}$. If $N$ is invariant by a group $G$ of isometries of $\mathrm{M}$ acting on TM by tangent maps, then such an $\mathcal{O} \subset N$ can be chosen to be $G$-invariant.

Proof. In the construction of $\mathcal{O}$ in (2.17) we can take each $U_{\alpha}$ small enough so that $W_{\alpha} \subset N$. This yields $\mathcal{O} \subset \mathcal{O}_{F} \cap N$.

Now let $h$ be an isometry of M. For all $s \in \mathbb{R}$ and all $z \in T \mathrm{M}$ we have $\pi\left(\Phi_{s}\left(h_{*}(z)\right)\right)=\pi\left(h_{*}\left(\Phi_{s}(z)\right)\right)=h\left(\pi\left(\Phi_{s}(z)\right)\right)$ and so $F\left(h_{*}(z)\right)=(h \times$ $h)(F(z))$. This shows in particular that $\mathcal{O}_{F}$ is $G$-invariant. Thus, if $\mathcal{O}=$ $\cup_{\alpha \in A} W_{\alpha} \subset \mathcal{O}_{F} \cap N$ is serrate so is the $G$-invariant set $\cup_{h \in G} h_{*} \mathcal{O} \subset \mathcal{O}_{F} \cap N$.

Proposition 3.2. With $G$ as in Proposition 3.1, $\mathbf{P}$ is $G$-invariant provided that it is defined in a $G$-invariant open serrate $\mathcal{O}$ containing $\mathrm{M}$.

Proof. By the uniqueness of the adapted product structure if $h$ is an isometry of $\mathbf{M}$ then $h_{*} \mathbf{P} h_{*}^{-1}$ is equal to $\mathbf{P}$ since it satisfies all the conditions for an adapted structure on $\mathcal{O}$, for $\Theta$ and $E$ are invariant by $h_{*}$. (This also can be seen using $F \circ h_{*}=(h \times h) \circ F$. $)$

Proposition 3.3. Let $G$ act freely on $(\mathrm{M}, g)$ by isometries. Let the adapted structure $\mathbf{P}$ be defined in the serrate open set $\mathcal{O} \subset T \mathrm{M}$ containing $\mathrm{M}$. Then for all $z \in \mathcal{O}$,

$$
\operatorname{dim}\left(\mathfrak{G}_{z}^{T \mathrm{M}}+\mathbf{P} \mathfrak{G}_{z}^{T \mathrm{M}}\right)=2 \operatorname{dim} G
$$

Proof. (Here it is not necessary that $\mathcal{O}$ be $G$-invariant but only that it be open and serrate.) Since the action is free, for any basis $\left\{\xi^{1}, \cdots, \xi^{\operatorname{dim} G}\right\}$ of $\mathfrak{G}$ the action vector fields $\xi_{\mathrm{M}}^{i}$ in $\mathrm{M}$ are point-wise linearly independent in all of M. Thus, if $z=0_{p} \in T_{p} \mathrm{M}$ for some $p \in \mathrm{M}$ the statement follows from Proposition 2.2 which states $\mathbf{P}(u)_{0_{p}}^{h}=(u)_{0_{p}}^{v}$ for all $u \in T_{p} \mathrm{M}$.

So, let $z \in \mathcal{O} \backslash \mathrm{M}$ and $\gamma$ the unit-speed geodesic on M with $y \dot{\gamma}(0)=z$ and $y>0$. The action vector fields $\xi_{\mathrm{M}}^{i}$ restrict to linearly independent Jacobi fields along $\gamma$. Since the +1 and -1 eigen-bundles intersect trivially it follows from Proposition 2.4 that the canonical extensions $\xi_{T \mathrm{M}}^{i}$ of the $\xi_{\mathrm{M}}^{i}$ satisfy on $\mathcal{L}_{\gamma} \cap \mathcal{O}$,

$$
\left[\xi_{T \mathrm{M}}^{1}\right]^{+} \wedge \cdots \wedge\left[\xi_{T \mathrm{M}}^{\operatorname{dim} G}\right]^{+} \wedge\left[\xi_{T \mathrm{M}}^{1}\right]^{-} \wedge \cdots \wedge\left[\xi_{T \mathrm{M}}^{\operatorname{dim} G}\right]^{-} \neq 0
$$


For if there were $a_{i} \in \mathbb{R}$ not all zero such that $0=\sum_{i=1}^{n} a_{i}\left[\xi_{T \mathrm{M}}^{i}(z)\right]^{+}$ then the Jacobi field along $\gamma$ given by the restriction to this geodesic of the action vector field $\zeta_{T \mathrm{M}}$ with $\zeta=\sum_{i=1}^{n} a_{i} \xi^{i} \in \mathfrak{G}$ would vanish at $\gamma(\|z\|)$ contradicting that the action is free. Similarly $0=\sum_{i=1}^{n} a_{i}\left[\xi_{T \mathrm{M}}^{i}(z)\right]^{-}$would imply that $\zeta_{T M}$ vanishes at $\gamma(-\|z\|)$.

Definition 3.1. Let $\mathbf{P}$ be defined in $\mathcal{O} \subset T \mathrm{M}$. We set

$$
H \stackrel{\text { def }}{=}\left(\mathfrak{G}^{T \mathrm{M}}+\mathbf{P} \mathfrak{G}^{T \mathrm{M}}\right)^{\perp}
$$

Proposition 3.4. $H$ and $H^{\perp}$ are $\mathbf{P}$-invariant. If $\mathcal{O} \subset T \mathrm{M}$ is $G$-invariant so are $H$ and $H^{\perp}$.

Proof. The $G$-invariance follows from the fact that $\mathbf{P}, d \Theta$ and the distribution $\mathfrak{G}^{T \mathrm{M}}$ are all $G$-invariant. On the other hand, since $d \Theta$ is anti-P-invariant, for any subspace $A \subset T_{z}(T \mathrm{M})$,

$$
\mathbf{P}\left(A^{\perp}\right)=(\mathbf{P} A)^{\perp}
$$

It follows that $H$ is $\mathbf{P}$-invariant since, obviously, so is $H^{\perp}=\mathfrak{G}^{T \mathrm{M}}+\mathbf{P} \mathfrak{G}^{T \mathrm{M}}$.

Proposition 3.5. With the notation as in Proposition 3.3, if in addition $\mathcal{O}$ is $G$-invariant, there is a $G$-invariant open set $\mathcal{O}^{\dagger} \subset \mathcal{O}$, containing $\mathrm{M}$, where $T(T \mathrm{M})$ splits in a $G$-invariant, $\mathbf{P}$-invariant and $d \Theta$-orthogonal way,

$$
\left.T(T \mathrm{M})\right|_{\mathcal{O}^{\dagger}}=H \oplus H^{\perp}
$$

Proof. By Proposition $3.3 \operatorname{dim} H^{\perp}=2 \operatorname{dim} G$ and since $d \Theta$ is non-degenerate as a two-form on $T \mathrm{M}$ we have $\operatorname{dim} H=2 n-2 \operatorname{dim} G$. Thus, at $z \in \mathcal{O} \subset T \mathrm{M}$ we have a splitting of $T_{z}(T \mathrm{M})=H_{z}^{\perp} \oplus H_{z}$ if and only if $H_{z} \cap H_{z}^{\perp}=\{0\}$, equivalently, if and only if $d \Theta$ is non-degenerate on $H_{z}^{\perp}=\mathfrak{G}_{z}^{T \mathrm{M}}+\mathbf{P} \mathfrak{G}_{z}^{T \mathrm{M}}$.

Given a basis of $\mathfrak{G},\left\{\xi^{1}, \cdots \xi^{\operatorname{dim} G}\right\}, d \Theta$ is non-degenerate on $\mathfrak{G}_{z}^{T \mathrm{M}}+\mathbf{P} \mathfrak{G}_{z}^{T \mathrm{M}}$ if and only $\operatorname{det} \Gamma(z) \neq 0$ for

$$
\Gamma(z) \stackrel{\operatorname{def}}{=}\left(\begin{array}{cc}
\left\{d \Theta\left(\xi_{T \mathrm{M}}^{i}(z), \xi_{T \mathrm{M}}^{j}(z)\right)\right\} & -\left\{d \Theta\left(\mathbf{P} \xi_{T \mathrm{M}}^{i}(z), \xi_{T \mathrm{M}}^{j}(z)\right)\right\} \\
\left\{d \Theta\left(\mathbf{P} \xi_{T \mathrm{M}}^{i}(z), \xi_{T \mathrm{M}}^{j}(z)\right)\right\} & -\left\{d \Theta\left(\xi_{T \mathrm{M}}^{i}(z), \xi_{T \mathrm{M}}^{j}(z)\right)\right\}
\end{array}\right),
$$


where the curl-brackets indicate $\operatorname{dim} G \times \operatorname{dim} G$ matrix blocks and where we used the anti-P-invariance of $d \Theta$. Since $\operatorname{det} \Gamma: \mathcal{O} \rightarrow \mathbb{R}$ is a continuous function, the set

$$
\mathcal{O}^{\dagger} \stackrel{\text { def }}{=}\{z \in \mathcal{O} \mid \operatorname{det} \Gamma(z) \neq 0\}
$$

is open. This set is also $G$-invariant for $d \Theta, \mathbf{P}$ and the distribution $\mathfrak{G}^{T \mathrm{M}}$ are all $G$-invariant. We now show that $\mathcal{O}^{\dagger}$ actually contains $\mathrm{M}$, that is,

$$
\forall x \in \mathrm{M}, \operatorname{det} \Gamma(x) \neq 0 .
$$

On the one hand, the determinants of the two off-diagonal blocks of $\Gamma(x)$ are not zero when $x \in \mathrm{M}$, for

$$
\begin{aligned}
\operatorname{det}\left\{d \Theta\left(\mathbf{P} \xi_{T \mathrm{M}}^{i}\left(0_{x}\right), \xi_{T \mathrm{M}}^{j}\left(0_{x}\right)\right)\right\} & =\operatorname{det}\left\{d \Theta\left(\mathbf{P}\left(\xi_{\mathrm{M}}^{i}(x)\right)_{0_{x}}^{h},\left(\xi_{\mathrm{M}}^{j}(x)\right)_{0_{x}}^{h}\right)\right\} \\
& =\operatorname{det}\left\{d \Theta\left(\left(\xi_{\mathrm{M}}^{i}(x)\right)_{0_{x}}^{v},\left(\xi_{\mathrm{M}}^{j}(x)\right)_{0_{x}}^{h}\right)\right\} \\
& =\operatorname{det}\left\{g\left(\xi_{\mathrm{M}}^{i}(x), \xi_{\mathrm{M}}^{j}(x)\right)\right\} \\
& \neq 0
\end{aligned}
$$

where we used: $\xi_{T \mathrm{M}}^{i}\left(0_{x}\right)=\left(\xi_{\mathrm{M}}^{i}(x)\right)_{0_{x}}^{h}$ which follows from our identification of $\mathrm{M}$ with the zero section; equation (2.5); the formula $d \Theta(U, V)=$ $g\left(\mathbf{K} U, \pi_{*} V\right)-g\left(\mathbf{K} V, \pi_{*} U\right)$; that $\left\{\xi^{1}, \cdots \xi^{\operatorname{dim} G}\right\}$ is a basis for $\mathfrak{G}$ and that the action if free.

On the other hand, every entry of the two diagonal blocks of $\Gamma(x)$ for $x \in \mathrm{M}$ vanish, since for all $1 \leq i, j \leq \operatorname{dim} G$ and for all $x \in \mathrm{M}$

$$
d \Theta\left(\xi_{T \mathrm{M}}^{i}\left(0_{x}\right), \xi_{T \mathrm{M}}^{j}\left(0_{x}\right)\right)=d \Theta\left(\left(\xi_{\mathrm{M}}^{i}(x)\right)_{0_{x}}^{h},\left(\xi_{\mathrm{M}}^{j}(x)\right)_{0_{x}}^{h}\right)=0 .
$$

But (3.8) and (3.9) implies (3.7), hence $\mathcal{O}^{\dagger} \supset \mathrm{M}$, and thus $\mathcal{O}^{\dagger}$ satisfies all the stated properties.

Corollary 3.5.1. On $\mu^{-1}(0) \cap \mathcal{O}^{\dagger}$ we have the $G$-invariant splitting

$$
\left.T\left(\mu^{-1}(0)\right)\right|_{\mu^{-1}(0) \cap \mathcal{O}^{\dagger}}=\mathfrak{G}^{T \mathrm{M}} \oplus H
$$

Thus, (3.10) defines a connection for the G-bundle $\mathfrak{p}: \mu^{-1}(0) \cap \mathcal{O}^{\dagger} \rightarrow T \mathrm{~B}$ whose horizontal distribution $H$ is $\mathbf{P}$-invariant. 
Proof. Recall the $G$-invariant and $\mathbf{P}$-invariant splitting $\left.T(T \mathrm{M})\right|_{\mathcal{O}^{\dagger}}=H^{\perp} \oplus$ $H$ from Proposition 3.5, and that $T\left(\mu^{-1}(0)\right)=\left(\mathfrak{G}^{T \mathrm{M}}\right)^{\perp}$. Consider:

i) on $\mathcal{O} \supset \mathcal{O}^{\dagger}, H \cap T\left(\mu^{-1}(0)\right)=H$, for $\mathfrak{G}^{T \mathrm{M}} \subset \mathfrak{G}^{T \mathrm{M}}+\mathbf{P} \mathfrak{G}^{T \mathrm{M}}=H^{\perp}$, hence $H \subset\left(\mathfrak{G}^{T \mathrm{M}}\right)^{\perp}$.

ii) on $\mu^{-1}(0) \cap \mathcal{O}^{\dagger}, \quad H^{\perp} \cap T\left(\mu^{-1}(0)\right)=\mathfrak{G}^{T \mathrm{M}}$, for $\mathbf{P} \mathfrak{G}^{T \mathrm{M}} \cap\left(\mathfrak{G}^{T \mathrm{M}}\right)^{\perp}=\{0\}$ on $\mathcal{O}^{\dagger}$, while $\mathfrak{G}^{T \mathrm{M}} \subset\left(\mathfrak{G}^{T \mathrm{M}}\right)^{\perp}$ on $\mu^{-1}(0)$.

From i) and ii) and counting dimensions we get on $\mu^{-1}(0) \cap \mathcal{O}^{\dagger}$ the $G$-invariant splitting (3.10), while clearly $\operatorname{ker} \mathfrak{p}_{*}=\mathfrak{G}^{T \mathrm{M}}$.

3.0.4. Proof of Theorem 2. By Corollary 3.5.1, the splitting $\left.T\left(\mu^{-1}(0)\right)\right|_{\mu^{-1}(0) \cap \mathcal{O}^{\dagger}}=\mathfrak{G}^{T \mathrm{M}} \oplus H$ is $G$-invariant with $H$, in addition, Pinvariant. Since at $z \in \mu^{-1}(0) \cap \mathcal{O}^{\dagger}$ we have ker $\mathfrak{p}_{*}=\mathfrak{G}_{z}^{T \mathrm{M}}$, it follows that $\left.\mathfrak{p}_{*}\right|_{H_{z}}: H_{z} \rightarrow T_{f_{*} z}(T \mathrm{~B})$ is a vector space isomorphism. So, we define $\check{\mathbf{P}}$ on $\mathfrak{p}\left(\mu^{-1}(0) \cap \mathcal{O}^{\dagger}\right) \subset T$ B so that for all $U \in H_{z}$,

$$
\check{\mathbf{P}}\left(\mathfrak{p}_{*} U\right)=\mathfrak{p}_{*}(\mathbf{P} U) .
$$

Because under the identification (3.4), $\left.\mathfrak{p}_{*}\right|_{H}$ preserves the one-forms $\Theta$ and $\Theta_{\mathrm{B}}$ as well as $d E$ and $d E_{\mathrm{B}}$, $\check{\mathbf{P}}$ satisfies on $\mathfrak{p}\left(\mu^{-1}(0) \cap \mathcal{O}^{\dagger}\right) \subset T \mathrm{~B}$,

$$
\check{\mathbf{P}}^{2}=\mathbf{I}, \quad \check{\mathbf{P}} d \Theta_{\mathrm{B}}=d E_{\mathrm{B}} .
$$

Now, let the adapted product structure $\mathbf{P}_{\mathrm{B}}$ corresponding to the submersion metric on $\mathrm{B}$ be defined in some serrate neighborhood of $\mathrm{B}, \mathcal{O}_{\mathrm{B}} \subset T \mathrm{~B}$. Since $\mathfrak{p}\left(\mu^{-1}(0) \cap \mathcal{O}^{\dagger}\right)$ is an open set in $T \mathrm{~B}$ containing $\mathrm{B}$, then, both $\check{\mathbf{P}}$ and $\mathbf{P}_{\mathrm{B}}$ are defined on

$$
\mathcal{O}_{\mathrm{B}}^{\prime} \subset \mathcal{O}_{\mathrm{B}} \cap \mathfrak{p}\left(\mu^{-1}(0) \cap \mathcal{O}^{\dagger}\right)
$$

which, by Proposition 3.1, can be assumed to be a serrate neighborhood of $\mathrm{B}$ in TB. In order to show that $\check{\mathbf{P}}=\mathbf{P}_{\mathrm{B}}$ on $\mathcal{O}_{\mathrm{B}}^{\prime}$, in light of (3.12) and our uniqueness result in Theorem 1 , it suffices to prove that $\check{\mathbf{P}}$ is integrable. The integrability of $\check{\mathbf{P}}$ in $\mathcal{O}_{\mathrm{B}}^{\prime} \subset T \mathrm{~B}$ follows if we show that for all local sections of $T(T \mathrm{~B}), U$ and $V$, defined in open sets of $\mathcal{O}_{\mathrm{B}} \supset \mathcal{O}_{\mathrm{B}}^{\prime}$, the Frobenius condition holds, i.e., there are local sections $W$ and $W^{\prime}$ such that

$$
[U+\check{\mathbf{P}} U, V+\check{\mathbf{P}} V] \stackrel{(i)}{=} W+\check{\mathbf{P}} W, \quad[U-\check{\mathbf{P}} U, V-\check{\mathbf{P}} V] \stackrel{(i i)}{=} W^{\prime}-\check{\mathbf{P}} W^{\prime}
$$

We now show this Frobenius condition for $\check{\mathbf{P}}$ using that it holds for $\mathbf{P}$. Recall that a vector field $X$ defined in an open set $\mathcal{O} \subset T \mathrm{M}$ is a local section of the bundle $H$ if and only if

$$
d \Theta\left(X, \xi_{T \mathrm{M}}\right) \stackrel{(*)}{=} 0, \quad d \Theta\left(X, \mathbf{P} \xi_{T \mathrm{M}}\right) \stackrel{(* *)}{=} 0, \quad \forall \xi \in \mathfrak{G} .
$$


Let $X_{1}$ and $X_{2}$ be local sections of $H$ and calculate

$$
\begin{aligned}
0=d^{2} \Theta\left(X_{1}, X_{2}, \xi_{T \mathrm{M}}\right) \stackrel{\dagger}{=} X_{1} \cdot\left(d \Theta\left(X_{2}, \xi_{T \mathrm{M}}\right)\right)+X_{2} \cdot\left(d \Theta\left(\xi_{T \mathrm{M}}, X_{1}\right)\right) \\
+\xi_{T \mathrm{M}} \cdot\left(d \Theta\left(X_{1}, X_{2}\right)\right)-d \Theta\left(\left[X_{1}, X_{2}\right], \xi_{T \mathrm{M}}\right)-d \Theta\left(\left[X_{2}, \xi_{T \mathrm{M}}\right], X_{1}\right) \\
\quad-d \Theta\left(\left[\xi_{T \mathrm{M}}, X_{1}\right], X_{2}\right) .
\end{aligned}
$$

The first two terms to the right of equality $(\dagger)$ vanish by $(*)$ of $(3.14)$, while the third, the fifth and the sixth cancel out, since the Lie derivative of $d \Theta$ along $\xi_{T \mathrm{M}}$ vanishes. Thus we get

$$
\text { (†) } d \Theta\left(\left[X_{1}, X_{2}\right], \xi_{T M}\right)=0 .
$$

Since $H$ is $\mathbf{P}$-invariant, $X_{1} \pm \mathbf{P} X_{1}$ and $X_{2} \pm \mathbf{P} X_{2}$ are also sections of $H$ and hence, putting $Y_{1}=\left[X_{1}+\mathbf{P} X_{1}, X_{2}+\mathbf{P} X_{2}\right]$ and $Y_{2}=\left[X_{1}-\mathbf{P} X_{1}, X_{2}-\mathbf{P} X_{2}\right]$ in $(\ddagger)$,

$$
0 \stackrel{* * *}{=} d \Theta\left(Y_{1}, \xi_{T \mathrm{M}}\right)=d \Theta\left(Y_{2}, \xi_{T \mathrm{M}}\right)
$$

as well; that is, $(*)$ of $(3.14)$ holds for $Y_{1}$ and $Y_{2}$. But (**) of (3.14) also holds for $Y_{1}$ and $Y_{2}$, which is seen by using in $(* * *)$ the anti-P-invariance of $d \Theta$ together with $\mathbf{P} Y_{1}=Y_{1}, \mathbf{P} Y_{2}=-Y_{2}$, true since the Frobenius condition is met for $\mathbf{P}$ which is integrable. We conclude:

$\left[X_{1}+\mathbf{P} X_{1}, X_{2}+\mathbf{P} X_{2}\right]$ and $\left[X_{1}-\mathbf{P} X_{1}, X_{2}-\mathbf{P} X_{2}\right]$ are local sections of $H$ whenever $X_{1}$ and $X_{2}$ are.

Now let $U$ and $V$ as in (3.13) be given. We will show the identity (i) of (3.13), (ii) being analogous.

Since $\left.\mathfrak{p}\right|_{\mu^{-1}(0) \cap \mathcal{O}^{\dagger}}: \mu^{-1}(0) \cap \mathcal{O}^{\dagger} \rightarrow \mathfrak{p}\left(\mu^{-1}(0) \cap \mathcal{O}^{\dagger}\right)$ is a principal $G$-bundle with connection whose horizontal distribution is $H$, there are unique " $H$ horizontal lifts" of $U$ and $V$. That is, there are "p-related" ( $G$-invariant in our case) local sections $X_{1}$ and $X_{2}$ of $H$ that project to the sections $U$ and $V$, i.e., $\mathfrak{p}_{*} X_{1}=U$ and $\mathfrak{p}_{*} X_{2}=V$. Applying definition (3.11) we see that $\mathbf{P} X_{1}$ and $\mathbf{P} X_{2}$ are the unique $H$-horizontal lifts for $\check{\mathbf{P}} U$ and $\check{\mathbf{P}} V$.

Since $\mathbf{P}$ is integrable in $\mathcal{O}$, there is a section $X_{3}$ such that

$$
\left[X_{1}+\mathbf{P} X_{1}, X_{2}+\mathbf{P} X_{2}\right]=X_{3}+\mathbf{P} X_{3} .
$$

Applying $\mathfrak{p}$ and (3.11) to the left side of equality (3.15) yields the left side of the equality (i) of (3.13). But, from what we showed a few lines above, the right side of equation (3.15) is a section of $H$, hence using (3.11),

$$
\check{\mathbf{P}}_{*}\left(X_{3}+\mathbf{P} X_{3}\right)=\mathfrak{p}_{*} \mathbf{P}\left(X_{3}+\mathbf{P} X_{3}\right)=\mathfrak{p}_{*}\left(X_{3}+\mathbf{P} X_{3}\right),
$$

so that $\mathfrak{p}_{*}\left(X_{3}+\mathbf{P} X_{3}\right)=W+\check{\mathbf{P}} W$ for a section $W$ as required in (3.13). 
3.0.5. Two examples. We illustrate some elements in Theorem 2, in particular $\mathcal{O}^{\dagger}$ vs. $\mathcal{O}$, with two examples.

1) In $\mathrm{M}=\mathbb{R}^{2} \times \mathbb{R}$ with the Euclidean metric $G=\mathbb{R}$ acts by

$$
(x, y, r) \stackrel{\theta}{\mapsto}(x \cos \theta-y \sin \theta, x \sin \theta+y \cos \theta, r+\theta),
$$

with

$\xi_{\mathrm{M}}(x, y, r)=-y \frac{\partial}{\partial x}+x \frac{\partial}{\partial y}+\frac{\partial}{\partial r}, \quad \xi_{T \mathrm{M}}(z)=-y \frac{\partial}{\partial x}+x \frac{\partial}{\partial y}+\frac{\partial}{\partial r}-v \frac{\partial}{\partial u}+u \frac{\partial}{\partial v}$,

where $(x, y, r, u, v, t))$ are the coordinates of the vector $z=u \frac{\partial}{\partial x}+v \frac{\partial}{\partial y}+t \frac{\partial}{\partial r} \in$ $T \mathrm{M} \cong \mathbf{R}^{6}$.

Here, $\mathbf{P} \frac{\partial}{\partial x}=\frac{\partial}{\partial u}, \mathbf{P} \frac{\partial}{\partial y}=\frac{\partial}{\partial v}$ and $\mathbf{P} \frac{\partial}{\partial r}=\frac{\partial}{\partial t}$, thus

$$
\mathbf{P} \xi_{T \mathrm{M}}(z)=-y \frac{\partial}{\partial u}+x \frac{\partial}{\partial v}+\frac{\partial}{\partial t}-v \frac{\partial}{\partial x}+u \frac{\partial}{\partial y}
$$

valid in $\mathcal{O}=T \mathrm{M}$. Note that $\xi_{T \mathrm{M}} \wedge \mathbf{P} \xi_{T \mathrm{M}} \neq 0$ throughout $T \mathrm{M}$.

Since $d \Theta=d u \wedge d x+d v \wedge d y+d t \wedge d r$,

$$
f(z) \stackrel{\text { def }}{=} d \Theta\left(\mathbf{P} \xi_{T \mathrm{M}}(z), \xi_{T \mathrm{M}}(z)\right)=x^{2}+y^{2}-v^{2}-u^{2}+1,
$$

and thus $\mathcal{O}^{\dagger}=\{z=(x, y, r, u, v, t) \in T \mathrm{M} \mid f(z) \neq 0\}$ is a $G$-invariant open set containing $\mathrm{M}=\{z=(x, y, r, 0,0,0) \in T \mathrm{M}\}$.

To describe the splitting of $T_{z}(T \mathrm{M})$ for $z \in \mathcal{O}^{\dagger}$ we use the global product coordinates $x^{ \pm}=x \pm u, y^{ \pm}=y \pm v$, and $r^{ \pm}=r \pm t$. Recall $U^{ \pm}=\frac{1}{2}(U \pm \mathbf{P} U)$. So $\left[\frac{\partial}{\partial x}\right]^{ \pm}=\frac{\partial}{\partial x^{ \pm}}$and $\left[\frac{\partial}{\partial u}\right]^{ \pm}= \pm \frac{\partial}{\partial x^{ \pm}}$. Similarly $\left[\frac{\partial}{\partial y}\right]^{ \pm}=\frac{\partial}{\partial y^{ \pm}},\left[\frac{\partial}{\partial v}\right]^{ \pm}= \pm \frac{\partial}{\partial y^{ \pm}}$, $\left[\frac{\partial}{\partial r}\right]^{ \pm}=\frac{\partial}{\partial r^{ \pm}}$and $\left[\frac{\partial}{\partial t}\right]^{ \pm}= \pm \frac{\partial}{\partial r^{ \pm}}$. Thus, $\left[\xi_{T M}(z)\right]^{ \pm}=-y^{ \pm} \frac{\partial}{\partial x^{ \pm}}+x^{ \pm} \frac{\partial}{\partial y^{ \pm}}+\frac{\partial}{\partial r^{ \pm}}$.

Let

$$
V_{1}^{+}=\frac{\partial}{\partial x^{+}}+y^{-} \frac{\partial}{\partial r^{+}}, \quad V_{1}^{-}=\frac{\partial}{\partial x^{-}}+y^{+} \frac{\partial}{\partial r^{-}}
$$

and

$$
V_{2}^{+}=\frac{\partial}{\partial y^{+}}-x^{-} \frac{\partial}{\partial r^{+}}, \quad V_{2}^{-}=\frac{\partial}{\partial y^{-}}-x^{+} \frac{\partial}{\partial r^{-}} .
$$

We check directly that on all $T \mathrm{M}$,

$$
\operatorname{span}\left\{V_{1}^{+}, V_{1}^{-}, V_{2}^{+}, V_{2}^{-}\right\}=\left(\operatorname{span}\left\{\left[\xi_{T \mathrm{M}}\right]^{-},\left[\xi_{T \mathrm{M}}\right]^{+}\right\}\right)^{\perp}
$$

and that the splitting $[T(T \mathrm{M})]^{ \pm}=\operatorname{span}\left\{V_{i}^{ \pm},\left[\xi_{T \mathrm{M}}\right]^{ \pm}\right\}$holds except whenever $\left[\xi_{T \mathrm{M}}\right]^{ \pm} \wedge V_{1}^{ \pm} \wedge V_{2}^{ \pm}=0$ which is equivalent to

$$
\operatorname{det}\left(\begin{array}{ccc}
-y^{ \pm} & x^{ \pm} & 1 \\
1 & 0 & y^{\mp} \\
0 & 1 & -x^{\mp}
\end{array}\right)=x^{ \pm} x^{\mp}+y^{ \pm} y^{\mp}+1 \equiv f(z)=0 .
$$


2) Consider the $S^{1}$-action on the standard sphere $S^{3}=\left\{\sum_{i=1}^{4} x_{i}^{2}=1\right\} \subset$ $\mathbb{R}^{4} \cong \mathbb{C}^{2}$ defining the Hopf fibration:

$$
\exp (\sqrt{-1} t) \cdot\left(w_{1}, w_{2}\right)=\left(\exp (\sqrt{-1} t) w_{1}, \exp (\sqrt{-1} t) w_{2}\right)
$$

where $w_{1}=x_{1}+\sqrt{-1} x_{2}$ and $w_{2}=x_{3}+\sqrt{-1} x_{4}$. This action is isometric and for $\xi=\frac{\partial}{\partial t} \in \mathfrak{s}(1)$,

$$
\xi_{S^{3}}=-x_{2} \frac{\partial}{\partial x_{1}}+x_{1} \frac{\partial}{\partial x_{2}}-x_{4} \frac{\partial}{\partial x_{3}}+x_{3} \frac{\partial}{\partial x_{4}} .
$$

Using the construction by Jacobi fields in Theorem 1, we have, for $z \in T S^{3}$, besides $\mathbf{P}(z)_{z}^{h}=(z)_{z}^{v}$,

$$
\mathbf{P}(u)_{z}^{h}=\|z\| \cot \|z\|(u)_{z}^{v}, \text { if } g(z, u)=0 .
$$

Moreover $\mathbf{P}$ is defined on $\mathcal{O}=\left\{z \in T S^{3} \mid g(z, z)<\frac{\pi}{2}\right\}$.

Choose $z=x_{3} \frac{\partial}{\partial x_{1}}-x_{4} \frac{\partial}{\partial x_{2}}-x_{1} \frac{\partial}{\partial x_{3}}+x_{2} \frac{\partial}{\partial x_{4}} \in T S^{3}$ and let $0<a<\frac{\pi}{2}$. Then $g\left(z, \xi_{S^{3}}\right)=g\left(\nabla_{z} \xi_{S^{3}}, z\right)=0$ and it follows, from $\xi_{T S^{3}}(a z)=\left(\xi_{S^{3}}(p)\right)_{a z}^{h}+$ $\left(\nabla_{a z} \xi_{S^{3}}\right)_{a z}^{v}$ where $p=\pi(z)$, and $\|z\|^{2}=1$, that

$$
\mathbf{P} \xi_{T S^{3}}(a z)=a \cot a\left(\xi_{S^{3}}(p)\right)_{a z}^{v}+a^{-1} \tan a\left(\nabla_{a z} \xi_{S^{3}}\right)_{a z}^{h} .
$$

Thus, since $\left\|\xi_{S^{3}}\right\|^{2}=1$ and $\left\|\nabla_{a z} \xi_{S^{3}}\right\|^{2}=a^{2}$,

$$
d \Theta\left(\mathbf{P} \xi_{T S^{3}}(a z), \xi_{T S^{3}}(a z)\right)=a(\cot a-\tan a),
$$

which vanishes at $a=\frac{\pi}{4}$. This shows $\mathcal{O}^{\dagger} \varsubsetneqq \mathcal{O}$. (In fact it holds that $\mathcal{O}^{\dagger}=$ $\mathcal{O} \backslash\left\{z \in T S^{3} \mid g(z, z)=\frac{\pi}{4}\right\}$, corresponding to the fact that the Riemannian quotient $S^{3} / S^{1}$ is the round two-sphere of radius $\frac{1}{2},\left\{\sum_{i=1}^{3} x_{i}^{2}=\frac{1}{4}\right\} \subset \mathbb{R}^{3}$. This is explained in general by the converse of Theorem 3 proven in [1].) We thank the referee for suggesting this example.

\section{Tangentially positive isometric actions.}

Definition 4.1. Since $d \Theta$ is anti-P-invariant wherever $\mathbf{P}$ is defined there is a Pseudo-Riemannian metric of signature $(n, n)$

$$
G_{\mathbf{P}}(U, V) \stackrel{\text { def }}{=} d \Theta(\mathbf{P} U, V)
$$


Remark 4.1. The metric $G_{\mathbf{P}}$ is natural with respect to symplectic reduction, that is "reduction commutes with submersion". For, if we have $\mu^{-1}(0) \cap \mathcal{O}^{*} \subset T \mathrm{M}$ and $\mathcal{O}_{\mathrm{B}}^{\prime} \subset T \mathrm{~B}$ as in the proof of Theorem 2, we have shown $G_{\mathbf{P}}$ restricts to a pseudo-Riemannian metric of signature $(n-\operatorname{dim} G, n)$ on $\mu^{-1}(0) \cap \mathcal{O}^{*}$ so that the pseudo-Riemannian submersion metric induced in the quotient coincides in $\mathcal{O}_{\mathrm{B}}^{\prime} \subset\left(\mu^{-1}(0) \cap \mathcal{O}^{*}\right) / G$ with the metric $G_{\mathbf{P}_{\mathrm{B}}}$ defined by $d \Theta_{\mathrm{B}}$ and $\mathbf{P}_{\mathrm{B}}$.

Proposition 4.1. Let $\mathrm{M} \subset \mathcal{S} \subset \mu^{-1}(0) \cap \mathcal{O}^{\dagger}$. If $\mathcal{S}$ is connected $G_{\mathbf{P}}$ is positive-definite on $\left.\mathfrak{G}^{T \mathrm{M}}\right|_{\mathcal{S}}$.

Proof. We have $T\left(\mu^{-1}(0)\right)=\left(\mathfrak{G}^{T \mathrm{M}}\right)^{\perp}$, hence with a choice of a basis for $\mathfrak{G}$,

$$
\Gamma(z)=\left(\begin{array}{cc}
\{0\} & -\left\{d \Theta\left(\mathbf{P} \xi_{T \mathrm{M}}^{i}(z), \xi_{T \mathrm{M}}^{j}(z)\right)\right\} \\
\left\{d \Theta\left(\mathbf{P} \xi_{T \mathrm{M}}^{i}(z), \xi_{T \mathrm{M}}^{j}(z)\right)\right\} & \{0\}
\end{array}\right)
$$

for all $z \in \mu^{-1}(0)$. If $S \subset \mu^{-1}(0) \cap \mathcal{O}^{\dagger}$ is connected, the signature of the (symmetric) lower left block of $\Gamma$ remains constant on $\mathcal{S}$. But, a calculation as in (3.8) shows that for all $x \in \mathrm{M}$

$$
\left\{d \Theta\left(\mathbf{P} \xi_{T \mathrm{M}}^{i}\left(0_{x}\right), \xi_{T \mathrm{M}}^{j}\left(0_{x}\right)\right)\right\}=\left\{g\left(\xi_{\mathrm{M}}^{i}(x), \xi_{\mathrm{M}}^{j}(x)\right)\right\},
$$

which is positive-definite.

Proposition 4.1 motivates the following

Definition 4.2. Tangentially positive action. Let $(\mathrm{M}, g)$ have the adapted structure $\mathbf{P}$ defined in $\mathcal{O} \subset T \mathrm{M}$ and let $\mathcal{S}$ be any subset of $\mathcal{O}$. We say that an isometric $G$-action on $\mathrm{M}$ is tangentially positive on $\mathcal{S}$ if and only if the pseudo-Riemannian metric

$$
G_{\mathbf{P}} \text { is positive-definite in }\left.\mathfrak{G}^{T \mathrm{M}}\right|_{\mathcal{S}} .
$$

Remark 4.2. In the definition, there is no a priori condition on the isometric action, such as being free. In fact, when $G$ acts trivially it acts tangentially positively on any set $\mathcal{S}$ where $\mathbf{P}$ is defined.

The reason for the definition is the following result, the converse of which we prove in [1]. 
Theorem 3. If ( $\mathrm{M}, g)$ has no conjugate points and $G$ acts isometrically on $\mathrm{M}$, freely, properly and tangentially positively on $\mu^{-1}(0)$, then the Riemannian quotient $\left(\mathrm{B}, g_{\mathrm{B}}\right)$ has no conjugate points.

Proof. By Proposition 2.5, if $\mathrm{M}$ has no conjugate points, in the proof of Theorem 2 we can take $\mathcal{O}=T \mathrm{M}$ as the set where $\mathbf{P}$ is defined. If in addition $G$ acts tangentially positively we can take $\mathcal{O}^{\dagger}=T \mathrm{M}$ also. Hence the reduction $\check{\mathbf{P}}$ of $P$, which is an adapted product structure, is defined on all $\mu^{-1}(0) / G=T$ B. Corollary 2.4.1 then implies that $\left(\mathrm{B}, g_{\mathrm{B}}\right)$ has no conjugate points as claimed. (Furthermore, the last statement and Proposition 2.5 then shows that $P_{\mathrm{B}}$ is defined on all $T \mathrm{~B}$, and hence, by Theorem $1, \check{\mathbf{P}}=\mathbf{P}_{\mathrm{B}}$.)

Remark 4.3. Since $\mu^{-1}(0)$ is connected and contains M, by Proposition 4.1, the positive-definiteness of $G_{\mathbf{P}}$ on $\left.\mathfrak{G}^{T \mathrm{M}}\right|_{\mu^{-1}(0)}$ in the statement of Theorem 3 is equivalent to the non-degeneracy of $d \Theta$ on $\left.\left(\mathfrak{G}^{T \mathrm{M}}+\mathbf{P} \mathfrak{G}^{T \mathrm{M}}\right)\right|_{\mu^{-1}(0)}$. Clearly not so for other subsets of TM.

4.0.6. An example. Let $\mathrm{H}^{n}=\left\{\left(x_{1}, \cdots, x_{n}\right) \mid x_{i} \in \mathbb{R}, X_{n}>0\right\}$ with the hyperbolic metric $g=\sum_{i=1}^{n} d x_{i}^{2} / x_{n}^{2}$. We have

$$
T \mathrm{H}^{n}=\left\{\left(x_{1}, \cdots, x_{n}, u_{1}, \cdots, u_{n}\right) \mid x_{i}, u_{i}, \in \mathbb{R}, x_{n} \in \mathbb{R}>0\right\}
$$

where $z=\left.\sum_{i=1}^{n} u_{i} \frac{\partial}{\partial x_{i}}\right|_{\left(x_{1}, \cdots x_{n}\right)}$ is identified with $\left(x_{1}, \cdots, x_{n}, u_{i}, \cdots, u_{n}\right)$.

Consider the isometric $\mathbb{R}$-action on $\mathrm{H}^{n}$

$$
t \cdot\left(x_{1}, \cdots, x_{n}\right)=\left(e^{t} x_{1}, \cdots, e^{t} x_{n}\right)
$$

Then

$$
\xi_{\mathrm{H}^{n}}\left(x_{1}, \cdots, x_{n}\right)=\sum_{i=1}^{n} x_{i} \frac{\partial}{\partial x_{i}}, \quad \xi_{T \mathrm{H}^{n}}(z)=\sum_{i=1}^{n}\left(x_{i} \frac{\partial}{\partial x_{i}}+u_{i} \frac{\partial}{\partial u_{i}}\right) .
$$

We now calculate $\mathbf{P} \xi_{T \mathrm{H}^{n}}(z)$ at $z \in \mu^{-1}(0)$.

Since $\mathrm{H}^{n}$ has constant sectional curvature equal to -1 we have, for example from the construction of $P$ via the Jacobi fields in (2.14), that

$$
\begin{aligned}
& \mathbf{P}(w)_{z}^{h}=\|z\| \operatorname{coth}\|z\|(w)_{z}^{v} \text { if } g(w, z)=0 ; \\
& \mathbf{P}(z)_{z}^{h}=(z)_{z}^{v},
\end{aligned}
$$

where $\|z\|^{2}=x_{n}^{-2} \sum_{i=1}^{n} u_{i}^{2}$. 
Now, we decompose $\xi_{T \mathrm{H}^{n}}(z)$ into horizontal and vertical components. We get, using $g\left(\nabla_{\frac{\partial}{\partial x_{i}}} \frac{\partial}{\partial x_{j}}, \frac{\partial}{\partial x_{k}}\right)=\frac{1}{2}\left(\frac{\partial g_{j k}}{\partial x_{i}}+\frac{\partial g_{i k}}{\partial x_{j}}-\frac{\partial g_{i j}}{\partial x_{k}}\right)$ for all $i, j, k$, with $\delta_{i j}$ the Kroenecker delta:

$$
\begin{aligned}
& \nabla_{\frac{\partial}{\partial x_{i}}} \frac{\partial}{\partial x_{j}}=\frac{\delta_{i j}}{x_{n}} \frac{\partial}{\partial x_{n}}, i \neq n \neq j \\
& \nabla_{\frac{\partial}{\partial x_{i}}} \frac{\partial}{\partial x_{n}}=\nabla_{\frac{\partial}{\partial x_{n}}} \frac{\partial}{\partial x_{i}}=-\frac{1}{x_{n}} \frac{\partial}{\partial x_{i}}, 1 \leq i \leq n .
\end{aligned}
$$

It follows that $\nabla_{\frac{\partial}{\partial x_{j}}} \xi_{\mathrm{H}^{n}}=\frac{x_{j}}{x_{n}} \frac{\partial}{\partial x_{n}}$ for $j \neq n$ and $\nabla_{\frac{\partial}{\partial x_{n}}} \xi_{\mathrm{H}^{n}}=-\sum_{i=1}^{n-1} \frac{x_{i}}{x_{n}} \frac{\partial}{\partial x_{i}}$.

Thus at $z=\sum_{i=1}^{n} u_{i} \frac{\partial}{\partial x_{i}} \in T_{p} \mathrm{H}^{n}$ with $p=\left(x_{1}, \cdots, x_{n}\right)$

$$
\begin{aligned}
\nabla_{z} \xi_{\mathrm{H}^{n}} & =-\frac{u_{n}}{x_{n}} \sum_{i=1}^{n} x_{i} \frac{\partial}{\partial x_{i}}+\left(\sum_{i=1}^{n} \frac{u_{i} x_{i}}{x_{n}^{2}}\right) x_{n} \frac{\partial}{\partial x_{n}} \\
& =-\frac{u_{n}}{x_{n}} \xi_{\mathrm{H}^{n}}(p)+\mu(z) x_{n} \frac{\partial}{\partial x_{n}}
\end{aligned}
$$

where $\mu(z)=x_{n}^{-2} \sum_{i=1}^{n} u_{i} x_{i}$ is the moment map of the action applied to the basis element $\xi=\frac{\partial}{\partial t}$ of the Lie Algebra of $\mathbb{R}$. Thus,

$$
\begin{aligned}
\xi_{T \mathrm{H}^{n}}(z) & =\left(\xi_{\mathrm{H}^{n}}(p)\right)_{z}^{h}+\left(\nabla_{z} \xi_{\mathrm{H}^{n}}\right)_{z}^{v} \\
& =\left(\xi_{\mathrm{H}^{n}}(p)\right)_{z}^{h}+\left(-\frac{u_{n}}{x_{n}} \xi_{\mathrm{H}^{n}}(p)+\mu(z) x_{n} \frac{\partial}{\partial x_{n}}\right)_{z}^{v} .
\end{aligned}
$$

Given $z \in \mu^{-1}(0) \cap T_{p} \mathbb{H}^{n}$, in addition to the vanishing of the obvious term in (4.5), we also have $g\left(\xi_{\mathrm{H}^{n}}(\pi z), z\right)=0$ and, since $g\left(\nabla_{z} \xi_{\mathrm{H}^{n}}, z\right)=0$, using $(4.4)$,

$$
\mathbf{P} \xi_{T \mathrm{H}^{n}}(z)=\|z\| \operatorname{coth}\|z\|\left(\xi_{\mathrm{H}^{n}}(p)\right)_{z}^{v}-\frac{\tanh \|z\|}{\|z\|} \frac{u_{n}}{x_{n}}\left(\xi_{\mathrm{H}^{n}}(p)\right)_{z}^{h} .
$$

It follows that at $z \in \mu^{-1}(0)$,

$$
\left.G_{\mathbf{P}}\left(\xi_{T \mathrm{H}^{n}}, \xi_{T \mathrm{H}^{n}}\right)\right|_{z}=\|z\|\left\|\xi_{\mathrm{H}^{n}}(\pi(z))\right\|^{2} \operatorname{coth}\|z\|\left(1-\frac{1}{\|z\|^{2}} \frac{u_{n}^{2}}{x_{n}^{2}} \tanh ^{2}\|z\|\right)
$$

which is always positive since $u_{n}^{2} / x_{n}^{2} \leq\|z\|^{2}$ and $\tanh ^{2}\|z\|<1$. Thus the $\mathbb{R}$-action (4.3) is tangentially positive on $\mu^{-1}(0)$.

Remark 4.4. The $\mathbb{R}$-action (4.3) is not tangentially positive on the whole $T \mathrm{H}^{n}$. To show this as concretely as possible, take $z=\left.a \sum_{i=1}^{n}\left(\frac{\partial}{\partial x_{i}}\right)\right|_{p}=$ 
$a \xi_{\mathrm{H}^{n}}(p) \notin \mu^{-1}(0)$ where $p=(1, \cdots, 1)$ and $0 \neq a \in \mathbb{R}$. Set in (4.5) $u_{i}=$ $x_{i}=1,1 \leq i \leq n$, then

$$
\xi_{T \mathrm{H}^{n}}(z)=\left(\xi_{\mathrm{H}^{n}}(p)\right)_{a \xi_{\mathrm{H}^{n}(p)}}^{h}+a\left(-\xi_{\mathrm{H}^{n}}(p)+n \frac{\partial}{\partial x_{n}}\right)_{a \xi_{\mathrm{H}^{n}(p)}}^{v} .
$$

By (4.4) and $\|z\|=\sqrt{n} a$,

$$
\mathbf{P} \xi_{T \mathrm{H}^{n}}(z)=\left(\xi_{\mathrm{H}^{n}}(p)\right)_{a \xi_{\mathrm{H}^{n}(p)}^{v}}^{v}+\frac{\tanh (a \sqrt{n})}{a \sqrt{n}}\left(-\xi_{\mathrm{H}^{n}}(p)+n \frac{\partial}{\partial x_{n}}\right)_{a \xi_{\mathrm{H}^{n}(p)}}^{h} .
$$

So, at the chosen $z$,

$$
\begin{aligned}
G_{\mathbf{P}}\left(\xi_{T \mathrm{H}^{n}}, \xi_{T \mathrm{H}^{n}}\right) & =\left\|\xi_{\mathrm{H}^{n}}(p)\right\|^{2}-\frac{a \tanh (a \sqrt{n})}{\sqrt{n}}\left\|-\xi_{\mathrm{H}^{n}}(p)+n \frac{\partial}{\partial x_{n}}\right\|^{2} \\
& =n-\frac{a \sqrt{n}(n-1)^{-1}}{\operatorname{coth}(a \sqrt{n})},
\end{aligned}
$$

which is negative if we let $a>>1$. (See [1] for more results along this line.)

\section{Stable and Unstable Jacobi fields under reduction.}

Let $(\mathrm{M}, g)$ be a complete Riemannian manifold with no conjugate points. The geodesic flow $\Phi$ on the unit tangent bundle $S \mathrm{M}=\{z \in T \mathrm{M} \mid g(z, z)=1\}$ is said to be of Anosov type if the orthogonal complement to the flow with respect to the Sasaki metric,

$$
G_{S}(U, V)=g\left(\pi_{*} U, \pi_{*} V\right)+g(\mathbf{K} U, \mathbf{K} V),
$$

splits into a sub-bundle of exponentially contracted and a sub-bundle of exponentially expanded directions under the action of the differential of the geodesic flow.

More specifically, put for $z \in S \mathrm{M}$

$$
W_{z}:=\left\{U \in T_{z}(S \mathrm{M}) \mid G_{S}(U, \Sigma(z))=0\right\},
$$

where $\Sigma$ is the geodesic spray, $\Sigma(z)=(z)_{z}^{h}$. Then, the geodesic flow is of Anosov type if and only if for each $z \in S M$ there are positive constants $a, b, c$, such that we have a splitting

$$
T_{z}(S \mathrm{M})=\mathbb{R} \Sigma(z) \oplus\left(W_{z}\right) \text { contract } \oplus\left(W_{z}\right)_{\text {coxpand }}^{\text {Anosov }}
$$


where $\left(W_{z}\right)_{\text {contract }}^{\text {Anoso }}$ consists of those vectors $U \in W_{z}$ with

$$
\left\|\left(\Phi_{t}\right)_{*} U\right\|_{G_{S}} \leq a\|U\|_{G_{S}} e^{-c t} \forall t>0, \quad\left\|\left(\Phi_{t}\right)_{*} U\right\|_{G_{S}} \geq b\|U\|_{G_{S}} e^{-c t} \forall t<0,
$$

and the $\left(W_{z}\right) \begin{gathered}\text { Anosov } \\ \text { expand }\end{gathered}$ is the $\left(W_{z}\right)$ contract for the reversed geodesic flow. Here $\|U\|_{G_{S}}$ is the length of $U$ in the Sasaki metric (5.1).

Remark 5.1. Hyperbolic space $\mathrm{H}^{n}$ is an example of a manifold with Anosov flow. In the previous section we showed that the $\mathbb{R}$-action (4.3) on $\mathrm{H}^{n}$ is tangentially positive on $\mu^{-1}(0)$. It turns out that the geodesic flow of $\mathrm{H}^{n} / \mathbb{R}=\mathrm{B}$ with the Riemannian submersion metric $g_{\mathrm{B}}$ is of Anosov type since $\mathrm{B}$ is isometric to $\mathrm{H}^{n-1}$ : In $\mathrm{H}^{n}$, with the notation as in paragraph containing (4.3), the vector field $\xi_{\mathrm{H}^{n}}$ together with $Y_{i}=x_{n} \frac{\partial}{\partial x_{i}}-x_{i} \frac{\partial}{\partial x_{n}}$ for $1 \leq i \leq n-1$ form a global frame for $T \mathbb{H}^{n}$. Moreover, $g\left(Y_{i}, \xi_{\mathrm{H}^{n}}\right)=0$ and $\left[Y_{i}, Y_{j}\right]=x_{j} \frac{\partial}{\partial x_{i}}-x_{i} \frac{\partial}{\partial x_{i}}$ for all $1 \leq i, j \leq n-1$. So $g\left(\left[Y_{i}, Y_{j}\right], \xi_{\mathrm{H}^{n}}\right)=0$ and the distribution of vectors orthogonal to the action is integrable. O'Neill's curvature formulas in [13] show that the quotient metric $g_{\mathrm{B}}$ has constant sectional curvature -1 .

Remark 5.1 motivates Theorem 4 below. To state the result we need to recall the definition of stable and unstable distributions by P. Eberlein in [4] for manifolds with no conjugate points (see also [5]). Given $z \in S \mathrm{M}$ and $0 \neq t \in \mathbb{R}$ let

$$
\mathbf{E}_{t}: T_{z}(T \mathrm{M}) \rightarrow T_{z}(T \mathrm{M})
$$

be the linear map such that for each $U \in T_{z}(T \mathrm{M})$ the image $\mathbf{E}_{t}(U) \in$ $T_{z}(T \mathrm{M})$ satisfies

$$
\pi_{*}\left(\mathbf{E}_{t}(U)\right)=\pi_{*} U, \quad \pi_{*}\left(\left(\Phi_{t}\right)_{*} \mathbf{E}_{t}(U)\right)=0 .
$$

Let

$$
\left(W_{z}\right)_{s-\text { stable }}^{\text {Jacoi }} \stackrel{\text { def }}{=}\left\{U \in W_{z} \mid \lim _{t \mapsto+\infty} \mathbf{E}_{t}(U)=U\right\}
$$

and

$$
\left(W_{z}\right)_{s-\text { unstable }}^{\text {Jacobi }} \stackrel{\text { def }}{=}\left\{U \in W_{z} \mid \lim _{t \mapsto-\infty} \mathbf{E}_{t}(U)=U\right\} .
$$

At each $z \in S \mathrm{M}$ the subspaces (5.5) and (5.6) have both dimension $n-1$, but they might intersect non-trivially. (See [4] for details.)

We will prove the following

Theorem 4. Let $(\mathrm{M}, g)$ be complete with no conjugate points. Let $G$ act on $\mathrm{M}$ by isometries, freely, properly, and tangentially positively on 
$\mu^{-1}(0)$, with $\left(\mathrm{B}=\mathrm{M} / G, g_{\mathrm{B}}\right)$ the Riemannian quotient. The distributions $(W)_{s-u n s t a b l e}^{J a c o b i}$ and $(W)_{s-s t a b l e}^{J a c o b i}$ on the unit tangent bundle $S \mathrm{M}$ can be symplectically reduced, and the reduced distributions agree with the distributions $\left(W_{\mathrm{B}}\right)_{s-\text { unstable }}^{J a c o b i}$ and $\left(W_{\mathrm{B}}\right)_{s-\text { stable }}^{\text {Jacobi }} \mathrm{SB}$.

If we let $(\mathrm{M}, g)$ have no conjugate points, by Theorem 1 , the adapted structure $\mathbf{P}$ is defined in all TM. This allows the introduction of a one-parameter family of re-scaled adapted product structures natural under reduction and whose \pm 1 -eigen-subbundles tend in the limit for the parameter going to $\infty$ to the strongly stable and strongly unstable Jacobi distributions. The proof of Theorem 4 is derived from Propositions 5.1 through 5.4 and comes right after Corollary 5.4.1.

Definition 5.1. Given $\lambda>0$, consider the multiplication by $\lambda$ along the fibers of $\pi: T \mathrm{M} \rightarrow \mathrm{M}$. Let $\lambda_{*}: T(T \mathrm{M}) \rightarrow T(T \mathrm{M})$ denote its tangent map and $\lambda^{*}: \Lambda^{*}(T \mathrm{M}) \rightarrow \Lambda^{*}(T \mathrm{M})$ the corresponding pull-back of forms, $\left(\lambda^{*} \omega\right)(U)=$ $\omega\left(\lambda_{*} U\right)$.

Put

$$
\mathbf{P}_{\lambda} \stackrel{\text { def }}{=} \lambda_{*}^{-1} \mathbf{P} \lambda_{*}
$$

Definition 5.2. Given a $\mathbf{P}_{\lambda}$-invariant subspace $A_{z} \subset T_{z}(T \mathrm{M})$ we put

$$
\left[A_{z}\right]_{\mathbf{P}_{\lambda}}^{ \pm} \stackrel{\text { def }}{=}\left\{U \pm \mathbf{P}_{\lambda} U \mid U \in A_{z}\right\}
$$

for clarity also we use the notation for the adapted structure $\mathbf{P}=\mathbf{P}_{1}$.

We also set,

$$
\left[A_{z}\right]_{\infty}^{ \pm} \stackrel{\text { def }}{=}\left\{U \in A_{z} \mid \lim _{\lambda \mapsto \infty}\left(U \mp \mathbf{P}_{\lambda} U\right)=0\right\} .
$$

Proposition 5.1. a) $\mathbf{P}_{\lambda}$ is integrable and $G$-invariant; b) $\mathbf{P}_{\lambda}$ is the unique product structure on $T \mathrm{M}$ with $\mathbf{P}_{\lambda} \Theta=\lambda^{-1} d E$.

Proof. For part a) we just note that $\mathbf{P}_{\lambda}$ is induced from the map $F_{\lambda}: T \mathrm{M} \rightarrow$ $\mathrm{M} \times \mathrm{M}$ given by $F_{\lambda}(z)=\left(\pi\left(\Phi_{-\lambda} z\right), \pi\left(\Phi_{\lambda} z\right)\right)$, hence is integrable and $G$ invariant. Equivalently, for $G$-invariance, note $\mathbf{P}$ is $G$-invariant and for any $\operatorname{map} h: \mathrm{M} \rightarrow \mathrm{M}, \lambda_{*}\left(h_{*}\right)_{*}=\left(h_{*}\right)_{*} \lambda_{*}$.

For part b) simply apply Theorem 1 to $\lambda_{*}\left(\mathbf{P}_{\lambda}\right) \lambda_{*}^{-1}$. 
Proposition 5.2. Given $0<\lambda \in \mathbb{R}$, the image of $W_{z}$ by map $\mathbf{E}_{t}: T_{z}(T \mathrm{M}) \rightarrow T_{z}(T \mathrm{M})$ in (5.3) satisfies

$$
\mathbf{E}_{t}\left(W_{z}\right)= \begin{cases}{\left[W_{z}\right]_{\mathbf{P}_{\lambda}}^{-}} & \text {if } t=\lambda \\ {\left[W_{z}\right]_{\mathbf{P}_{\lambda}}^{+}} & \text {if } t=-\lambda .\end{cases}
$$

Proof. Fix $z \in S \mathrm{M}$ and let $\gamma$ be the unit speed geodesic with $\dot{\gamma}(0)=z$. For $U \in W_{z}$ let $J^{(t, U)}$ be the Jacobi field along $\gamma$ with conditions

$$
J^{(t, U)}(0)=\pi_{*} U, \quad J^{(t, U)}(t)=0 .
$$

Let $\tilde{J}^{(t, U)}(s, y)=\left(J^{(t, U)}(s)\right)_{y \dot{\gamma}(s)}^{h}+y\left(\nabla_{\dot{\gamma}(s)} J^{(t, U)}(s)\right)_{y \dot{\gamma}(s)}^{v}$ be the canonical extension of that Jacobi field along the leaf $\mathcal{L}_{\gamma}$ with parametrization $(s, y) \in$ $\mathbb{R}^{2} \mapsto y \dot{\gamma}(s) \in \mathcal{L}_{\gamma}$, so that the given $z=(0,1)$. Then

$$
\mathbf{E}_{t}(U)=\tilde{J}^{(t, U)}(0,1) .
$$

But, from the identities listed ahead in (5.20), it follows that

$$
\lambda_{*}(\tilde{J}(s, y))=\tilde{J}(s, \lambda y),
$$

and thus, we re-write (5.11) as

$$
\mathbf{E}_{t}(U)=\lambda_{*}^{-1}\left(\tilde{J}^{(t, U)}(0, \lambda)\right) .
$$

Consider $t=\lambda$. By (5.10) $J^{(\lambda, U)}(\lambda)=0$, hence by Proposition 2.4,

$$
\left[\tilde{J}^{(\lambda, U)}(0, \lambda)\right]_{\mathbf{P}}^{+}=0
$$

and thus, putting $t=\lambda$ in (5.13),

$$
\begin{aligned}
\mathbf{E}_{\lambda}(U) & =\lambda_{*}^{-1}\left(\left[\tilde{J}^{(\lambda, U)}(0, \lambda)\right]_{\mathbf{P}}^{-}\right) \\
& =\lambda_{*}^{-1}\left(\left[\lambda_{*}\left(\mathbf{E}_{\lambda}(U)\right)\right]_{\mathbf{P}}^{-}\right) \\
& =\left[\mathbf{E}_{\lambda}(U)\right]_{\mathbf{P}_{\lambda}}^{-} .
\end{aligned}
$$

Now, consider $t=-\lambda$. We have $J^{(-\lambda, U)}(-\lambda)=0$ by (5.10), and by Proposition 2.4

$$
\left[\tilde{J}^{(-\lambda, U)}(0, \lambda)\right]_{\mathbf{P}}^{-}=0 \text {. }
$$


Hence, setting $t=-\lambda$ in $(5.13)$,

$$
\begin{aligned}
\mathbf{E}_{-\lambda}(U) & =\lambda_{*}^{-1}\left(\left[\tilde{J}^{(-\lambda, U)}(0, \lambda)\right]_{\mathbf{P}}^{+}\right) \\
& =\lambda_{*}^{-1}\left(\left[\lambda_{*}\left(\mathbf{E}_{-\lambda}(U)\right)\right]_{\mathbf{P}}^{+}\right) \\
& =\left[\mathbf{E}_{-\lambda}(U)\right]_{\mathbf{P}_{\lambda}}^{+} .
\end{aligned}
$$

Equalities (5.14) and (5.15) show that $\mathbf{E}_{ \pm \lambda}\left(T_{z}(T \mathrm{M})\right) \subset\left[T_{z}(T \mathrm{M})\right]_{\mathbf{P}_{\lambda}}^{\mp} \cdot(\operatorname{In}$ fact, we have equalities since $\operatorname{dim} \mathbf{E}_{t}\left(T_{z}(M)\right)=n$. This is because $\mathrm{M}$ has no conjugate points and thus the subspace

$$
\left.\operatorname{ker} \mathbf{E}_{t}\right|_{z}=\left.\operatorname{ker} \pi_{*}\right|_{z}=\left\{U=(u)_{z}^{v} \mid u \in T_{\pi z} \mathrm{M}\right\} \subset T_{z}(T \mathrm{M}),
$$

which has dimension $n$.) But, for any $0 \neq t \in \mathbb{R}$,

$$
\mathbf{E}_{t}\left(W_{z}\right) \subset W_{z}
$$

for if $U \in W_{z}$, the Jacobi field $J^{(t, U)}$ defined by (5.10) along the geodesic $\gamma$ with $\dot{\gamma}(0)=z$ is normal to $\gamma$, hence, its canonical extension $\tilde{J}^{(t, U)}$, satisfies $d \Theta\left(\tilde{J}^{(t, U)}, \Sigma\right)=d \Theta\left(\tilde{J}^{(t, U)}, \Xi\right)=0$, that is $\tilde{J}^{(t, U)} \in W$. Thus, it follows, since $W_{z}$ is $\mathbf{P}_{\lambda}$-invariant, that

$$
\mathbf{E}_{ \pm \lambda}\left(W_{z}\right) \subset\left[W_{z}\right]_{\mathbf{P}_{\lambda}}^{\mp} .
$$

Equality in 5.17 follows if we show that $0 \neq t \in \mathbb{R}, \operatorname{dim} \mathbf{E}_{t}\left(W_{z}\right)=n-1$. But, $\operatorname{dim} W_{z}=2 n-2$ and $\operatorname{dim} W_{z} \cap \operatorname{ker} \mathbf{E}_{t}=\operatorname{dim}\left\{U=(u)_{z}^{v} \mid u \in T_{\pi z} \mathrm{M}, g(u, z)=\right.$ $0\}=n-1$.

Corollary 5.2.1. Given $\|z\|=1$, with notation as in (5.5), (5.6) and (5.8),

$$
\left[W_{z}\right]_{\infty}^{-}=\left(W_{z}\right)_{s-\text { stable }}^{\text {Jacobi }}, \quad\left[W_{z}\right]_{\infty}^{+}=\left(W_{z}\right)_{s-\text { unstable }}^{\text {Jacobi }}
$$

Proof. Let $z \in S \mathrm{M}$. If $U \in\left(W_{z}\right)_{s-\text { stable }}^{\text {Jacobi }}$ then $\lim _{\lambda \mapsto \infty} \mathbf{E}_{\lambda}(U)=U$. Thus, by Proposition 5.2 and (5.8), $U \in\left[W_{z}\right]_{\infty}^{-}$. Hence $\left(W_{z}\right)_{s-s t a b l e}^{J a c o b i} \subset\left[W_{z}\right]_{\infty}^{-}$. But equality holds since

$$
n-1=\operatorname{dim}\left(W_{z}\right)_{s-\text { stable }}^{\text {Jacobi }} \leq \operatorname{dim}\left[W_{z}\right]_{\infty}^{-} \leq n-1 .
$$

Similarly for $\left[W_{z}\right]_{\infty}^{+}$. 
Proposition 5.3. For any $0<\lambda \in \mathbb{R}$, the distribution on $T \mathrm{M} \backslash \mathrm{M}$

$$
W \stackrel{\text { def }}{=}(\mathbb{R} \Sigma+\mathbb{R} \Xi)^{\perp},
$$

and the distribution on $T \mathrm{M}$

$$
H^{\lambda} \stackrel{\text { def }}{=}\left(\mathfrak{G}^{T \mathrm{M}}+\mathbf{P}_{\lambda} \mathfrak{G}^{T \mathrm{M}}\right)^{\perp}
$$

are both $G$-invariant and $\mathbf{P}_{\lambda}$-invariant.

Proof. These distributions are $G$-invariant for $d \Theta$ and $\mathbf{P}_{\lambda}$ are $G$-invariant.

Furthermore, we have for all $z \in T \mathrm{M}$ and all $u \in T_{\pi(z)} \mathrm{M}$,

$$
\lambda_{*}\left((u)_{z}^{h}\right)=(u)_{\lambda z}^{h}, \quad \lambda_{*}\left((u)_{z}^{v}\right)=\lambda(u)_{\lambda z}^{v}=(\lambda u)_{\lambda z}^{v} .
$$

From (5.20), $\lambda^{*} d \Theta=\lambda d \Theta$, thus for any subspace $A \subset T_{z}(T \mathrm{M})$ it holds $\lambda_{*}\left(A^{\perp}\right)=\left(\lambda_{*} A\right)^{\perp}$. Hence, since $d \Theta$ is anti-P-invariant,

$$
\mathbf{P}_{\lambda}\left(A^{\perp}\right)=\left(\mathbf{P}_{\lambda} A\right)^{\perp} .
$$

But (5.21) implies that $W$ is $\mathbf{P}_{\lambda}$-invariant, for, from (5.20),

$$
\lambda_{*}(\Sigma(z))=\Sigma(\lambda z), \quad \lambda_{*}(\Xi(z))=\lambda \Xi(\lambda z),
$$

hence $\mathbf{P}_{\lambda} \Xi=\lambda \Sigma$, which implies that $W^{\perp}=\mathbb{R} \Sigma+\mathbb{R} \Xi=\mathbb{R} \Sigma+\mathbb{R} \mathbf{P}_{\lambda} \Sigma$ is $\mathbf{P}_{\lambda \text {-invariant. }}$

Similarly (5.21) implies the $\mathbf{P}_{\lambda}$-invariance of $H^{\lambda}$.

Corollary 5.3.1. Given any $0<\lambda \in \mathbb{R}$, we have the splitting

$$
T\left(\mu^{-1}(0)\right)=\mathfrak{G}^{T \mathrm{M}} \oplus H^{\lambda}
$$

which defines a connection for the $G$-bundle $\mathfrak{p}: \mu^{-1}(0) \rightarrow T$ B.

Proof. Recall the $d \Theta$-orthogonal $G$-invariant splitting $T\left(\mu^{-1}(0)\right)=\mathfrak{G}^{T \mathrm{M}} \oplus H$ that defines a connection with horizontal distribution $H=(\mathfrak{G}+\mathbf{P} \mathfrak{G})^{\perp}$ from Corollary 3.5.1.

Clearly multiplication by $\lambda$ along the fibers of $\pi: T \mathrm{M} \rightarrow \mathrm{M}$ acts on $\mu^{-1}(0) \subset T \mathrm{M}$. Now, by (5.20), for all $\xi \in \mathfrak{G}$ the action vector field $\xi_{T \mathrm{M}}(z)=$ $\left(\xi_{\mathrm{M}}\right)_{z}^{h}+\left(\nabla_{z} \xi_{\mathrm{M}}\right)_{z}^{v}$ re-scales as $\lambda_{*}\left(\xi_{T \mathrm{M}}(z)\right)=\xi_{T \mathrm{M}}(\lambda z)$, and thus

$$
\lambda_{*} \mathfrak{G}^{T \mathrm{M}}=\mathfrak{G}^{T \mathrm{M}} .
$$


So, since $\lambda^{*} d \Theta=\lambda d \Theta$, it follows that the splitting

$$
T\left(\mu^{-1}(0)\right)=\mathfrak{G}^{T \mathrm{M}} \oplus \lambda_{*}^{-1} H
$$

is $d \Theta$-orthogonal and $G$-invariant; it defines a connection with horizontal distribution

$$
\begin{aligned}
\lambda_{*}^{-1} H & =\lambda_{*}^{-1}\left(\left(\mathfrak{G}^{T \mathrm{M}}+\mathbf{P} \mathfrak{G}^{T \mathrm{M}}\right)^{\perp}\right) \\
& =\left(\lambda_{*}^{-1}\left(\mathfrak{G}^{T \mathrm{M}}+\mathbf{P} \mathfrak{G}^{T \mathrm{M}}\right)\right)^{\perp}=\mathfrak{G}^{T \mathrm{M}}+\mathbf{P}_{\lambda} \mathfrak{G}^{T \mathrm{M}}=H^{\lambda}
\end{aligned}
$$

Remark 5.2. $(\mathbb{R} \Xi+\mathbb{R} \Sigma)^{\perp}=\left\{U \in T(T \mathrm{M}) \mid G_{S}(U, \Sigma)=G_{S}(U, \Xi)=0\right\}$ since $G_{S}(U, \Xi)=g(\mathbf{K} U, z)=d \Theta(U, \Sigma)$, and $G_{S}(U, \Sigma)=g\left(\pi_{*} U, z\right)=$ $-d \Theta(U, \Xi)$. Thus (5.18) simply extends the definition of $W_{z}$ for $z \in S \mathrm{M}$ given in (5.2).

Proposition 5.4. Give $\mathrm{B}=\mathrm{M} / G$ the Riemannian metric $g_{\mathrm{B}}$ which makes $f: \mathrm{M} \rightarrow \mathrm{B}$ a Riemannian submersion. Let $\mathbf{P}_{\mathrm{B}}$ be the adapted structure defined on $T \mathrm{~B}$ and $W_{\mathrm{B}}=\left(\mathbb{R} \Sigma_{\mathrm{B}}+\mathbb{R}_{\mathrm{B}}\right)^{\perp}$ with $\Xi_{\mathrm{B}}$ and $\Sigma_{\mathrm{B}}$ respectively the Liouville vector field and the geodesic spray on $T \mathrm{~B}$.

Given $0<\lambda \in \mathbb{R}$, consider the total space of the principal $G$-bundle $\mathfrak{p}:\left(\mu^{-1}(0)\right) \rightarrow T \mathrm{~B}$ with the connection defined by $H^{\lambda}$. Then, at each $z \in \mu^{-1}(0)$, with notation as in (5.7) applied to $\mathbf{P}_{\lambda}$ and $\left(\mathbf{P}_{\mathrm{B}}\right)_{\lambda}=\lambda_{*}^{-1} \mathbf{P}_{\mathrm{B}} \lambda_{*}$,

$$
\mathfrak{p}_{*}:\left[H_{z}^{\lambda} \cap W_{z}\right]_{\mathbf{P}_{\lambda}}^{ \pm} \rightarrow\left[\left(W_{\mathrm{B}}\right)_{\mathfrak{p}_{*} z}\right]_{\left(\mathbf{P}_{\mathrm{B}}\right)_{\lambda}}^{ \pm}
$$

is an isomorphism.

Proof. For all $z \in \mu^{-1}(0)$, identifying as in (3.3),

$$
\mathfrak{p}_{*}:\left(H_{z}^{\lambda}, \Theta, E\right) \rightarrow\left(T_{\mathfrak{p}_{*} z} \mathrm{~B}, \Theta_{\mathrm{B}}, E_{\mathrm{B}}\right)
$$

is an isomorphism. Thus, for all $U \in H^{\lambda}$,

$$
d E(U)=d \Theta(U, \Sigma)=d \Theta_{\mathrm{B}}\left(\mathfrak{p}_{*} U, \mathfrak{p}_{*} \Sigma\right)=d E_{\mathrm{B}}\left(\mathfrak{p}_{*} U\right),
$$

and also,

$$
\Theta(U)=d \Theta(\Xi, U)=d \Theta_{\mathrm{B}}\left(\mathfrak{p}_{*} \Xi, \mathfrak{p}_{*} U\right)=\Theta_{\mathrm{B}}\left(\mathfrak{p}_{*} U\right),
$$


which, by the non-degeneracy of the symplectic forms, shows that

$$
\mathfrak{p}_{*} \Sigma=\Sigma_{\mathrm{B}}, \quad \mathfrak{p}_{*} \Xi=\Xi_{\mathrm{B}}
$$

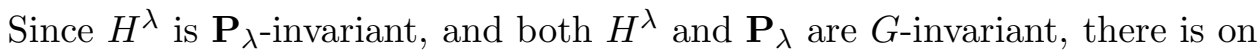
$T$ B a structure $\left(\mathbf{P}_{\lambda}\right)^{\sim}$ defined by

$$
\left(\mathbf{P}_{\lambda}\right)^{\curlyvee}\left(\mathfrak{p}_{*} U\right)=\mathfrak{p}_{*} \mathbf{P}_{\lambda} U
$$

for all $U \in H^{\lambda}$. Such reduced $\left(\mathbf{P}_{\lambda}\right)^{\sim}$ is integrable and squared gives the identity. Also,

$$
\Theta_{\mathrm{B}}\left(\left(\mathbf{P}_{\lambda}\right)^{\sim}\left(\mathfrak{p}_{*} U\right)\right)=\Theta_{\mathrm{B}}\left(\mathfrak{p}_{*}\left(\mathbf{P}_{\lambda} U\right)\right)=\Theta\left(\mathbf{P}_{\lambda} U\right)=\lambda d E(U)=\lambda d E_{\mathrm{B}}\left(\mathfrak{p}_{*} U\right),
$$

and thus $\left(\mathbf{P}_{\lambda}\right)^{\sim} \Theta_{\mathrm{B}}=\lambda d E_{\mathrm{B}}$, which implies, by Proposition 5.1 applied to $T \mathrm{~B}$,

$$
\left(\mathbf{P}_{\lambda}\right)^{-}=\left(\mathbf{P}_{\mathrm{B}}\right)_{\lambda}=\lambda_{*}^{-1} \mathbf{P}_{\mathrm{B}} \lambda_{*}
$$

From (5.25), (5.26), (5.27), $W=(\mathbb{R} \Sigma+\mathbb{R} \Xi)^{\perp}=\left(\mathbb{R} \Sigma+\mathbb{R} \mathbf{P}_{\lambda} \Sigma\right)^{\perp}$ and correspondingly $W_{\mathrm{B}}=\left(\mathbb{R} \Sigma_{\mathrm{B}}+\mathbb{R} \Xi_{\mathrm{B}}\right)^{\perp}=\left(\mathbb{R} \Sigma_{\mathrm{B}}+\mathbb{R}\left(\mathbf{P}_{\mathrm{B}}\right)_{\lambda} \Sigma_{\mathrm{B}}\right)^{\perp}$, it follows that for $z \in \mu^{-1}(0)$,

$$
\mathfrak{p}_{*}:\left(H_{z}^{\lambda} \cap W_{z}, \mathbf{P}_{\lambda}\right) \rightarrow\left(\left(W_{\mathrm{B}}\right)_{\mathfrak{p}_{*} z},\left(\mathbf{P}_{\mathrm{B}}\right)_{\lambda}\right)
$$

is an isomorphism, hence $\mathfrak{p}_{*}:\left[H_{z}^{\lambda} \cap W_{z}\right]_{\mathbf{P}_{\lambda}}^{ \pm} \rightarrow\left[\left(W_{\mathrm{B}}\right)_{\mathfrak{p}_{*} z}\right]_{\left(\mathbf{P}_{\mathrm{B}}\right)_{\lambda}}^{ \pm}$is an isomorphism.

Corollary 5.4.1. For each $z \in \mu^{-1}(0) \cap S \mathrm{M}$, in terms of Definition 5.2,

$$
\begin{aligned}
& \mathfrak{p}_{*}: \lim _{\lambda \mapsto \infty}\left[H_{z}^{\lambda} \cap W_{z}\right]_{\mathbf{P}_{\lambda}}^{-} \rightarrow\left(\left(W_{\mathrm{B}}\right)_{\mathfrak{p}_{*} z}\right)_{\text {s-stable }}^{\text {Jacobi }} \\
& \mathfrak{p}_{*}: \lim _{\lambda \mapsto \infty}\left[H_{z}^{\lambda} \cap W_{z}\right]_{\mathbf{P}_{\lambda}}^{+} \rightarrow\left(\left(W_{\mathrm{B}}\right)_{\mathfrak{p}_{*} z}\right)_{\text {s-unstable }}^{\text {Jacobi }} .
\end{aligned}
$$

are isomorphisms.

Proof. By Theorem 2, the Riemannian quotient $\left(\mathrm{B}, g_{\mathrm{B}}\right)$ has no conjugate points, and thus, by the paragraph following (5.6) applied to $\left(\mathrm{B}, g_{\mathrm{B}}\right)$, we know that $\operatorname{dim}\left(\left(W_{\mathrm{B}}\right)_{\mathfrak{p}_{*} z}\right)_{s-\text { stable }}^{\text {Jacobi }}=\operatorname{dim}\left(\left(W_{\mathrm{B}}\right)_{\mathfrak{p}_{*} z}\right)_{s-\text { unstable }}^{\text {Jacobi }}=\operatorname{dim} \mathrm{B}-1$. So, in light of Corollary 5.2.1 applied to $\left(\mathrm{B}, g_{\mathrm{B}}\right),\left[\left(W_{\mathrm{B}}\right)_{\mathfrak{p}_{*} z}\right]_{\infty}^{ \pm}$are well-defined subspaces of $T_{\mathfrak{p}_{*} z}(T \mathrm{~B})$ of dimension $\operatorname{dim} \mathrm{B}-1$. The Corollary follows by taking $\lambda \mapsto \infty$ in (5.24). 
5.0.7. Proof of Theorem 4. By Corollary 5.2.1, the limits as $\lambda \mapsto \infty$ of the direct summands in the splitting $W=[W]_{\mathbf{P}_{\lambda}}^{-} \oplus[W]_{\mathbf{P}_{\lambda}}^{+}$are the distributions we claim are being reduced. Thus, by Corollary 5.4.1, the theorem is proved if we show that on $\mu^{-1}(0) \cap S \mathrm{M}$

$$
\begin{aligned}
W \cap T\left(\mu^{-1}(0)\right) & =\mathfrak{G}^{T \mathrm{M}} \oplus\left(H^{\lambda} \cap W\right) \\
& =\mathfrak{G}^{T \mathrm{M}} \oplus\left[H^{\lambda} \cap W\right]_{\mathbf{P}_{\lambda}}^{-} \oplus\left[H^{\lambda} \cap W\right]_{\mathbf{P}_{\lambda}}^{+},
\end{aligned}
$$

which means that

$$
[W]_{\mathbf{P}_{\lambda}}^{ \pm} \cap T\left(\mu^{-1}(0)\right)=\left[H^{\lambda} \cap W\right]_{\mathbf{P}_{\lambda}}^{ \pm}
$$

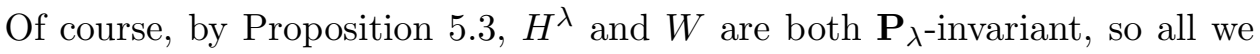
need to show is the first equality in (5.30), which we do next.

Since $W^{\perp}=\mathbb{R} \Sigma+\mathbb{R} \Xi$ and $d \Theta(\Xi, \Sigma)=2 E \neq 0$ off $\mathrm{M}$, we have $W \cap W^{\perp}=$ $\{0\}$ on $T \mathrm{M} \backslash \mathrm{M}$, and thus

$$
\left.T(T \mathrm{M})\right|_{T \mathrm{M} \backslash \mathrm{M}}=W^{\perp} \oplus W .
$$

The $d \Theta$-orthogonal splitting (5.31) is $G$-invariant, since $\Xi, \Sigma$ and $d \Theta$ are all $G$-invariant, and it is also $\mathbf{P}_{\lambda}$-invariant by the arguments in Proposition 5.3.

Now, by Proposition 5.22,

$$
T\left(\mu^{-1}(0)\right)=\mathfrak{G}^{T \mathrm{M}} \oplus H^{\lambda},
$$

where $H^{\lambda}=\left(\mathfrak{G}^{T \mathrm{M}}+\mathbf{P}_{\lambda} \mathfrak{G}^{T \mathrm{M}}\right)^{\perp}$. So, by (5.31), restricting to $\mu^{-1}(0) \backslash \mathrm{M}$ we have the $G$-invariant and $\mathbf{P}_{\lambda}$-invariant splitting

$$
\begin{aligned}
T & \left.\left(\mu^{-1}(0)\right)\right|_{\mu^{-1}(0) \backslash \mathrm{M}} \\
= & \underbrace{\left(\mathfrak{G}^{T \mathrm{M}} \cap W^{\perp}\right)}_{=\{0\}} \oplus \underbrace{\left(H^{\lambda} \cap W^{\perp}\right)}_{=W^{\perp}} \oplus \underbrace{\left(\mathfrak{G}^{T \mathrm{M}} \cap W\right)}_{=\mathfrak{G}^{T \mathrm{M}}} \oplus\left(H^{\lambda} \cap W\right) .
\end{aligned}
$$

To justify the equalities underlying the summands above, note that on $\mu^{-1}(0)$

$$
\mathfrak{G}^{T \mathrm{M}} \subset W,
$$

since for all $\xi \in \mathfrak{G}$ it holds $d \Theta\left(\xi_{T \mathrm{M}}(z), \Sigma(z)\right)=g\left(\xi_{\mathrm{M}}(\pi(z)), z\right)=0$ whenever $z \in \mu^{-1}(0)$, and $d \Theta\left(\xi_{T \mathrm{M}}(z), \Xi(z)\right)=-g\left(\nabla_{z} \xi_{\mathrm{M}}, z\right)=0$ by skew-symmetry of 
$\nabla \xi_{\mathrm{M}}$. But inclusion (5.32) implies $\mathfrak{G}^{T \mathrm{M}} \cap W=\mathfrak{G}^{T \mathrm{M}}$, as well as $\mathfrak{G}^{T \mathrm{M}} \cap W^{\perp}=$ $\{0\}$ in $\mu^{-1}(0) \backslash \mathrm{M}$, since there $W \cap W^{\perp}=\{0\}$; it also implies

$$
W^{\perp} \subset\left(\mathfrak{G}^{T \mathrm{M}}\right)^{\perp}=T\left(\mu^{-1}(0)\right),
$$

which forces $W^{\perp} \cap H^{\lambda}=W^{\perp}=\mathbb{R} \Xi+\mathbb{R} \Sigma$.

Thus we have shown

$$
T\left(\mu^{-1}(0)\right)=(\mathbb{R} \Xi+\mathbb{R} \Sigma) \oplus \underbrace{\mathfrak{G}^{T \mathrm{M}} \oplus\left(H^{\lambda} \cap W\right)}_{=W \cap T\left(\mu^{-1}(0)\right)},
$$

as required.

This section ends with two corollaries. To state the first one we recall from [4] the following

Definition 5.3. A Riemannian manifold $\left(\mathrm{B}, g_{\mathrm{B}}\right)$ is compactly homogeneous if there is a compact set $C \subset \mathrm{B}$ such that $\mathrm{B}=\cup_{h \in \text { Isometry }(\mathrm{B})} h(C)$.

Corollary 5.4.2. Let $(\mathrm{M}, g)$, be complete, with no conjugate points, and with sectional curvatures $\mathcal{K} \geq-c^{2}$, for a constant $c>0$. Also, assume that ( $\left.\mathrm{B}=\mathrm{M} / G, g_{\mathrm{B}}\right)$ is compactly homogeneous with $G$ as in Theorem 4. Then, if the geodesic flow of $(\mathrm{M}, g)$ is Anosov so is the geodesic flow of $\left(\mathrm{B}=\mathrm{M} / G, g_{\mathrm{B}}\right)$.

Proof. For any $U \in T(T \mathrm{M})$, from (5.1),

$$
g\left(\pi_{*}\left(\left(\Phi_{t}\right)_{*} U\right), \pi_{*}\left(\left(\Phi_{t}\right)_{*} U\right)\right) \leq G_{S}\left(\left(\Phi_{t}\right)_{*} U,\left(\Phi_{t}\right)_{*} U\right) .
$$

In particular, if $U$ is in $\left(W_{z}\right)_{\text {contract }}^{\text {Anosov }}$ the right-hand side of (5.33) is bounded above for all $t \geq 0$. Hence, if $\mathcal{K} \geq-c^{2}$, Proposition 2.12 in [4] implies that for the metric $g,\left(W_{z}\right)_{\text {contract }}^{\text {Anosov }} \subset\left(W_{z}\right)_{s-\text { stable }}^{\text {Jacobi }}$; and similarly, $\left(W_{z}\right)_{\text {expand }}^{\text {Anosov }} \subset$ $\left(W_{z}\right)_{\text {s-unstable }}^{\text {Jacobi }}$.

So $\left(W_{z}\right)_{\text {expand }}^{\text {Anosov }}=\left(W_{z}\right)_{s-\text { unstable }}^{\text {Jacobi }}$ and $\left(W_{z}\right)_{\text {contract }}^{\text {Annosov }}=\left(W_{z}\right)_{s-\text { stable }}^{\text {Jacobi }}$. Thus, if the flow of $g$ is of Anosov type,

$$
W=W_{s-\text { unstable }}^{J a c o b i} \oplus W_{s-\text { stable }}^{\text {Jacobi }},
$$

and by our Theorem 4 ,

$$
\left(W_{\mathrm{B}}\right)=\left(W_{\mathrm{B}}\right)_{\text {s-unstable }}^{\text {Jacobi }} \oplus\left(W_{\mathrm{B}}\right)_{\text {s-stable }}^{J a c o b i}
$$

Hence, from the assumption on $\left(\mathrm{B}, g_{\mathrm{B}}\right)$ and Theorem 3.2 in [4] the corollary is proved. 
Corollary 5.4.3. Let $(\mathrm{M}, g)$ be complete, with no conjugate points, with sectional curvatures $\mathcal{K} \geq-c^{2}$ for a constant $c>0$, and with geodesic flow of Anosov type. Let $G \subset$ Isom (M) act freely, properly and tangentially positively on $\mu^{-1}(0)$, with $\mathrm{M} / G$ a smooth compact manifold. If $N(G)$ is the normalizer of $G$ in Isom $(\mathrm{M})$, then $N(G) / G$ is finite.

Proof. The group $N(G)=\{h \in$ Isom (M) $\mid h G=G h\}$ acts on B $=\mathrm{M} / G$ via the correspondence $N(G) \ni h \mapsto \breve{h}$ where $\breve{h}(G \cdot p)=G \cdot h(p)$ for all $p \in \mathrm{M}$.

Such action is by isometries for the Riemannian submersion metric $g_{\mathrm{B}}$ corresponding to $f: \mathrm{M} \rightarrow \mathrm{B}$. For, if $h \in \operatorname{Isom}(\mathrm{M})$ then $\check{h} \circ f=f \circ h$, and $h$ preserves the $f$-horizontal distribution defined as the $g$-orthogonal complement to the fibers of $f$; thus if $u$ and $v$ in $T_{p} \mathrm{M}$ are $f$-horizontal we have, using the definition of the metric $g_{\mathrm{B}}, g_{\mathrm{B}}\left(\breve{h}_{*}\left(f_{*} u\right), \breve{h}_{*}\left(f_{*} v\right)\right)=$ $g_{\mathrm{B}}\left(f_{*}\left(h_{*} u\right), f_{*}\left(h_{*} v\right)\right)=g\left(h_{*} u, h_{*} v\right)=g(u, v)=g_{\mathrm{B}}\left(f_{*} u, f_{*} v\right)$. It follows that $\breve{h}$ is an isometry for $g_{\mathrm{B}}$.

But since $G$ is the kernel of the homomorphism $N(G) \rightarrow$ Isom (B) given by $h \mapsto \breve{h}$, the corollary follows from Theorem 4 and the result of W. Klingenberg in the appendix of [9] which states that the isometry group of a compact Riemannian manifold whose geodesic flow is Anosov must be finite.

\section{Final Remarks.}

The results obtained in this paper for actions by groups $G$ of isometries of arbitrary dimension acting on $\mathrm{M}$ tangentially positively in $\mu^{-1}(0)$ extend the results previously well-known for the case $\operatorname{dim} G=0$. Although when $(\mathrm{M}, g)$ is compact with negative-definite Ricci tensor, or compact with Anosov type geodesic flow, the isometry group is finite, for more general Riemannian manifolds, especially for non-compact ones, the question of whether there are groups of isometries of positive dimension acting tangentially positively is meaninful, as the $\mathbb{R}$-action (4.3) on $\mathrm{H}^{n}$ shows.

In light of Theorem 1, and due to the curvature decreasing property of submersions, one should expect restrictions on the existence of such actions. We hint here at such constraints, and develop more results along these lines in [1]. The next Proposition is actually used in [1].

Proposition 6.1. Let $G$ act by isometries on $\left(\mathbb{R}^{n}\right.$, Euclidean $)$, tangentially positively on $\mu^{-1}(0) \subset T \mathbb{R}^{n}$. Then, 
1) the fibers of the action on $\mathrm{M}=\mathbb{R}^{n}$ are hyper-planes;

2) if $G$ is Abelian, it acts by translations.

Proof. For $\xi \in \mathfrak{G}, p \in \mathrm{M}, z \in T_{p} \mathrm{M}$, and $a \in \mathbb{R}$, we have $\xi_{T \mathrm{M}}(a z)=$ $\left(\xi_{\mathrm{M}}(p)\right)_{a z}^{h}+a\left(\nabla_{z} \xi_{\mathrm{M}}\right)_{a z}^{v}, \mathbf{P} \xi_{T \mathrm{M}}(a z)=\left(\xi_{\mathrm{M}}(p)\right)_{a z}^{v}+a\left(\nabla_{z} \xi_{\mathrm{M}}\right)_{a z}^{h}$, and thus,

$$
d \Theta\left(\mathbf{P} \xi_{T \mathrm{M}}(z), \xi_{T \mathrm{M}}(z)\right)=\left\|\xi_{\mathrm{M}}(p)\right\|^{2}-a^{2}\left\|\nabla_{z} \xi_{\mathrm{M}}\right\|^{2}
$$

But $\mathbb{R} z \subset \mu^{-1}(0)$ provided that $z \in \mu^{-1}(0)$. It follows from the hypothesis on the action that $\left\|\xi_{\mathrm{M}}(p)\right\|^{2}-a^{2}\left\|\nabla_{z} \xi_{\mathrm{M}}\right\|^{2} \geq 0$ for all $a \in \mathbb{R}$. Thus,

$$
\nabla_{z} \xi_{\mathrm{M}}=0 \text { if } z \in \mu^{-1}(0) .
$$

Now part 1) follows from that given $\xi$ and $\zeta$ in $\mathfrak{G}$ and $z \in T_{p} \mathrm{M} \cap \mu^{-1}(0)$ we have

$$
g\left(\nabla_{\zeta_{\mathrm{M}}(p)} \xi_{\mathrm{M}}, z\right)=-g\left(\nabla_{z} \xi_{\mathrm{M}}, \zeta_{\mathrm{M}}\right)=0
$$

where the first equality uses skew-symmetry of $\nabla \xi_{\mathrm{M}}$ and the second one (6.1). Since $z$ is arbitrary in the $g$-orthogonal complement of $\mathfrak{G}_{p}^{M}$, then $\nabla_{\zeta_{\mathrm{M}}(p)} \xi_{\mathrm{M}}$ lies in $\mathfrak{G}_{p}^{M}$. This shows that the fibers are totally geodesic submanifolds.

To prove part 2) we first show that for any given $\xi \in \mathfrak{G}$

$$
\nabla_{\xi_{\mathrm{M}}} \xi_{\mathrm{M}}=0 \text { on } \mathrm{M}
$$

Let $p \in \mathrm{M}$ and $z \in T_{p} \mathrm{M}$. By (6.1) we already know that $g\left(\nabla_{\xi_{\mathrm{M}}(p)} \xi_{\mathrm{M}}, z\right)=0$ if $z \in \mu^{-1}(0)$. So we now take $z=\zeta_{\mathrm{M}}(p)$ for $\zeta \in \mathfrak{G}$. But

$$
g\left(\nabla_{\xi_{\mathrm{M}}} \xi_{\mathrm{M}}, \zeta_{\mathrm{M}}\right) \stackrel{(i)}{=}-g\left(\nabla_{\zeta_{\mathrm{M}}} \xi_{\mathrm{M}}, \xi_{\mathrm{M}}\right) \stackrel{(i i)}{=}-g\left(\nabla_{\xi_{\mathrm{M}}} \zeta_{\mathrm{M}}+\left[\zeta_{\mathrm{M}}, \xi_{\mathrm{M}}\right], \xi_{\mathrm{M}}\right) \stackrel{(i i i)}{=} 0
$$

where (i) uses skew-symmetry of $\nabla \xi_{\mathrm{M}}$, (ii) that the connection is torsionfree, and (iii) both the skew-symmetry of $\nabla \zeta_{\mathrm{M}}$ and that $G$ is Abelian, and thus (6.2) is proved.

Now, let $\xi \in \mathfrak{G}$ and $X$ a local vector field in a neighborhood of $p$ in M. 
Letting $\mathbf{R}_{\mathrm{M}}(X, Y)=\left[\nabla_{X}, \nabla_{Y}\right]-\nabla_{[X, Y]}$ be the curvature operator on $\mathrm{M}$,

$$
\begin{aligned}
\overbrace{g\left(\mathbf{R}_{\mathrm{M}}\left(X, \xi_{\mathrm{M}}\right) \xi_{\mathrm{M}}, X\right)}^{(a)} & =g \overbrace{\nabla_{X} \nabla_{\xi_{\mathrm{M}} \xi_{\mathrm{M}}}}^{\equiv 0 \text { by }}-\nabla_{\xi_{\mathrm{M}}} \nabla_{X} \xi_{\mathrm{M}}-\nabla_{\left[X, \xi_{\mathrm{M}}\right]} \xi_{\mathrm{M}}, X) \\
& =\overbrace{-g\left(\nabla_{\xi_{\mathrm{M}}} \nabla_{X} \xi_{\mathrm{M}}, X\right)}^{(b)} \overbrace{-g\left(\nabla_{\left[X, \xi_{\mathrm{M}}\right]} \xi_{\mathrm{M}}, X\right)}^{(c)}=(b) \\
& \stackrel{(*)}{=} \overbrace{-\nabla_{\xi_{\mathrm{M}}}\left(g\left(\nabla_{X} \xi_{\mathrm{M}}, X\right)\right)+g\left(\nabla_{X} \xi_{\mathrm{M}}, \nabla_{\xi_{\mathrm{M}}} X\right)}^{(b)}
\end{aligned}
$$

$(c)$

$$
\begin{aligned}
\overbrace{-g\left(\nabla_{\left[X, \xi_{\mathrm{M}}\right]} \xi_{\mathrm{M}}, X\right)} \\
\stackrel{(* *)}{=}\left|\nabla_{X} \xi_{\mathrm{M}}\right|^{2} \geq 0,
\end{aligned}
$$

where equality $(*)$ holds since $\nabla_{\xi_{\mathrm{M}}} g \equiv 0$. To see $(* *)$ note that the first term to the right of equality $\left(^{*}\right)$ is zero due to the skew-symmetry of $\nabla \xi_{\mathrm{M}}$; moreover, the second term to the right of $\left(^{*}\right)$ is re-written by using the vanishing of the torsion of $\nabla$ and again, the skew-symmetry of $\nabla \xi_{\mathrm{M}}$, this last property also used to re-write the term (c) and cancel it out with a term coming from (b).

Thus, if $\mathrm{M}$ is Euclidean $(a) \equiv 0$, we have,

$$
0=g\left(\mathbf{R}_{\mathrm{M}}\left(z, \xi_{\mathrm{M}}(p)\right) \xi_{\mathrm{M}}(p), z\right)=\left|\nabla_{z} \xi_{\mathrm{M}}\right|^{2} \geq 0
$$

It follows from (6.1) and (6.4) that $\nabla_{z} \xi_{\mathrm{M}}=0$ for all $z \in T_{p} \mathrm{M}$.

Remark 6.1. The definition of $\mathbf{P}$ in terms of the symplectic geometry of $T \mathrm{M}$ is inspired by the comparison of the adapted complex structure of $L$. Lempert and R. Szöke in [11] with the construction of the equivalent complex structure in the cotangent bundle by V. Guillemin and M. Stenzel in [6] by means of analytic continuation, hence the name adapted product structure.

Remark 6.2. In [1] we prove the converse of Theorem 3, showing that the "Pseudo-Riemannian reduction" fails precisely when conjugate points are created, allowing several applications.

6.0.8. Acknowledgements. We are thankful to the anonymous referee for helpful suggestions and corrections in an earlier version of the manuscript. 


\section{References.}

[1] Aguilar, R. Tangentially positive isometric actions and Conjugate Points. Preprint 2003. To appear.

[2] Aguilar, R. Symplectic reduction and the complex homogeneous MongeAmpère equation. Ann. Glob. Analysis \& Geom. 19 (2001), 327-353.

[3] Dombrowski, P. On the geometry of the tangent bundle. J. Reine Angew. Math 210 (1961), 73-88.

[4] Eberlein, P.: When is a geodesic flow of Anosov type? J.Differential Geom. 8 (1973), 437-463.

[5] Green, L. A theorem of E. Hopf. Mich. Math. J. (1958), 31-34.

[6] Guillemin, V. \& Stenzel, M.: Grauert tubes and the homogeneous Monge-Ampère equation I. J. Diff. Geometry, 34 (1991), 561-570.

[7] Helgason, S. Differential Geometry, Lie groups and symmetric spaces.(1978) Academic Press, New York.

[8] Hitchin, N., Karlhede, A., Linstrtröm, U., Roček, M.: Hyper-Kähler metrics and supersymmetry. Comm. Math. Phys. 108 (4) (1987), 535589 .

[9] Klingenberg, W.:Riemannian manifolds with geodesic flows of Anonosov type. Ann. of Math. 99 (1974), 1-13.

[10] Klingenberg, W. Riemannian Geometry. (1982) de Gruyter, Berlin.

[11] Lempert, L. \& Szőke, R.:Global solutions of the homogeneous complex Monge-Ampère equation and complex structures on the tangent bundle of Riemannian manifolds. Math. Ann. 290 (1991), 689-712.

[12] Marsden, J. \& Weisntein, A.:Reduction of symplectic manifolds with symmetry. Rep. Math. Phys. 5(1974), 121-130.

[13] O'Neill, B.:The fundamental equations of a submersion. Mich. Math. J. 13 (1966), 459-469.

Mathematics and Science Department, Massachusetts Maritime ACADEMY, BuZzards BAy, MA 02532

raguilar@maritime.edu

Received January 14, 2003. 